\title{
THE SHARP CONSTANT IN THE REVERSE HÖLDER INEQUALITY FOR MUCKENHOUPT WEIGHTS
}

\author{
V. VASYUNIN
}

\begin{abstract}
Coifman and Fefferman proved that the "reverse Hölder inequality" is fulfilled for any weight satisfying the Muckenhoupt condition. In order to illustrate the power of the Bellman function technique, Nazarov, Volberg, and Treil showed (among other things) how this technique leads to the reverse Hölder inequality for the weights satisfying the dyadic Muckenhoupt condition on the real line. In this paper the proof of the reverse Hölder inequality with sharp constants is presented for the weights satisfying the usual (rather than dyadic) Muckenhoupt condition on the line. The results are a consequence of the calculation of the true Bellman function for the corresponding extremal problem.
\end{abstract}

In [1] Coifman and Fefferman proved that for any weight (i.e., for any nonnegative function) $w$ on $\mathbb{R}^{n}$ satisfying the Muckenhoupt condition

$$
\sup _{Q \subset \mathbb{R}^{n}}\left\{\langle w\rangle_{Q}\left\langle w^{\frac{1}{1-p}}\right\rangle_{Q}^{p-1}\right\}<\infty
$$

(the supremum is taken over all cubes $Q$ with edges parallel to the coordinate axes) the "reverse Hölder inequality" is fulfilled:

$$
\left\langle w^{q}\right\rangle_{Q} \leq C\langle w\rangle_{Q}^{q}
$$

for some $q>1$, with a constant $C$ independent of the cube $Q$. Here and later, $\langle\varphi\rangle_{Q}$ stands for the average of the function $\varphi$ over the set $Q$ :

$$
\langle f\rangle_{Q} \stackrel{\text { def }}{=} \frac{1}{|Q|} \int_{Q} \varphi(t) d t, \quad|Q| \stackrel{\text { def }}{=} \int_{Q} d t .
$$

In 2 Nazarov, Treil, and Volberg illustrated the power of the Bellman function technique. In particular, they deduced the reverse Hölder inequality for dyadic $A_{\infty}$-weights on the real line by constructing a Bellman function. In the present paper we refine this technique to prove the reverse Hölder inequality for arbitrary $A_{p}$-weights $(1 \leq p \leq \infty)$ on the real line with a sharp constant $C$ depending on $p$ and $q$ (the exponent in the reverse Hölder inequality), and on the $A_{p^{-}}$"norm" $\delta$ of $w$. Furthermore, we find the sharp bound for the exponents $q$ for which the reverse Hölder inequality is true. Instead of Muckenhoupt's original definition of $A_{\infty}$-weights (used, e.g., in [1]), we employ the equivalent definition introduced by Khrushchëv [3]. Namely, the symbol $A_{\infty}^{\delta}(J)$ will denote the set of all nonnegative functions $w \in L^{1}(J)$ such that

$$
\sup _{I \subset J}\left\{\langle w\rangle_{I} \exp \left(-\langle\log w\rangle_{I}\right)\right\} \leq \delta<\infty .
$$

2000 Mathematics Subject Classification. Primary 42B20, 42B25.

Key words and phrases. Reverse Hölder inequality, Muckenhoupt weights, Bellman function.

Partially supported by RFBR (grant no. 02-01-00260a). 
Here $J$ and $I$ are intervals on the real line $\mathbb{R}$. Condition (1) is the limit case (as $p \rightarrow \infty$ ) of the $A_{p}$-condition

$$
\sup _{I \subset J}\left\{\langle w\rangle_{I}\left\langle w^{1-p^{\prime}}\right\rangle_{I}^{p-1}\right\} \leq \delta<\infty, \quad \frac{1}{p}+\frac{1}{p^{\prime}}=1 .
$$

In the other limit case $(p=1)$, we get the $A_{1}$-condition, which will be written in the form

$$
\sup _{I \subset J}\left\{\langle w\rangle_{I} \sup _{t \in I} \frac{1}{w(t)}\right\} \leq \delta<\infty .
$$

The set of all nonnegative functions $w \in L^{1}(J)$ satisfying (2)-(3) will be denoted by $A_{p}^{\delta}(J)$.

To become familiar with the general ideology of the Bellman function technique, the reader may address not only the paper [2] mentioned above, but also the earlier survey 4 by Nazarov and Treil. The role of the Muckenhoupt condition and the reverse Hölder inequality in the theory of singular integrals is described in any book concerning this subject, for example, in the monograph "Harmonic Analysis" by Stein [5], or in the survey [6] by Dyn'kin and Osilenker.

To state the main result of the paper, we need some more notation. For $p>1$ we introduce two functions $u_{p}^{ \pm}$as the functions inverse to

$$
t \mapsto(1-t)\left(1-\frac{t}{p}\right)^{-p}
$$

(to $t \mapsto(1-t) e^{t}$ for $p=\infty$ ) and defined on the following domains: $u_{p}^{+}:[0,1] \rightarrow[0,1]$, $u_{p}^{-}:[0,1] \rightarrow[-\infty, 0]$. In other words, the values $u_{p}^{ \pm}(t)$ are two solutions (the positive and the negative) of the equation $(1-u)(1-u / p)^{-p}=t, 0 \leq t \leq 1$.

For $1<p<\infty$, the Hölder inequality $\left\langle w_{1}^{p}\right\rangle^{1 / p}\left\langle w_{2}^{p^{\prime}}\right\rangle^{1 / p^{\prime}} \geq\left\langle w_{1} w_{2}\right\rangle$ implies that $\delta \geq 1$ for the constant in (2). For $p=\infty$, the same estimate for the constant $\delta$ in (1) follows from the Jensen inequality $\left\langle e^{\varphi}\right\rangle \geq e^{\langle\varphi\rangle}$. Therefore, for $p>1$ we can define $s^{ \pm}=s_{p}^{ \pm}(\delta) \stackrel{\text { def }}{=}$ $u_{p}^{ \pm}(1 / \delta)$. Finally, we introduce yet another parameter, putting $\gamma=\gamma(p, q) \stackrel{\text { def }}{=} \frac{p q-q+1}{p}$.

Now we state the main result of the paper.

Theorem 1. For any weight $w$ in $A_{p}^{\delta}(J), 1<p<\infty$, the following inequalities are true:

$$
C_{\min }(p, q, \delta)\langle w\rangle_{J}^{q} \leq\left\langle w^{q}\right\rangle_{J} \leq C_{\max }(p, q, \delta)\langle w\rangle_{J}^{q},
$$

where the constants

$$
\begin{aligned}
& C_{\min }(p, q, \delta) \\
& = \begin{cases}1, & q \in(-\infty, 0] \cup[1,+\infty), \\
\delta^{\frac{1-q}{p}} \frac{\left(1-s^{+}\right)^{\gamma}}{1-\gamma s^{+}}, & q \in[0,1],\end{cases} \\
& C_{\max }(p, q, \delta) \\
& = \begin{cases}+\infty, & q \in\left(-\infty, \frac{p-s^{-}}{s^{-}(p-1)}\right] \cup\left[\frac{p-s^{+}}{s^{+}(p-1)},+\infty\right), \\
\delta^{\frac{1-q}{p}} \frac{\left(1-s^{+}\right)^{\gamma}}{1-\gamma s^{+}}, & q \in\left[-\frac{1}{p-1}, 0\right] \cup\left[1, \frac{p-s^{+}}{s^{+}(p-1)}\right), \\
\delta^{\frac{1-q}{p}} \frac{\left(1-s^{-}\right)^{\gamma}}{1-\gamma s^{-}}, & q \in\left(\frac{p-s^{-}}{s^{-}(p-1)},-\frac{1}{p-1}\right], \\
1, & q \in[0,1],\end{cases}
\end{aligned}
$$

are sharp. 
We state the limit cases of Theorem 1 for $p=\infty$ and $p=1$ separately.

Theorem $\mathbf{1}_{\infty}$. For any weight $w$ in $A_{\infty}^{\delta}(J)$, inequalities (4) are true with the sharp constants

$$
\begin{aligned}
C_{\min }(\infty, q, \delta)= \begin{cases}1, & q \in(-\infty, 0] \cup[1,+\infty), \\
\frac{\left(1-s^{+}\right)^{q}}{1-q s^{+}}, & q \in[0,1],\end{cases} \\
C_{\max }(\infty, q, \delta)= \begin{cases}+\infty, & q \in\left(-\infty, \frac{1}{s^{-}}\right] \cup\left[\frac{1}{s^{+}},+\infty\right), \\
\frac{\left(1-s^{+}\right)^{q}}{1-q s^{+}}, & q \in\left[1, \frac{1}{s^{+}}\right), \\
\frac{\left(1-s^{-}\right)^{q}}{1-q s^{-}}, & q \in\left(\frac{1}{s^{-}}, 0\right], \\
1, & q \in[0,1] .\end{cases}
\end{aligned}
$$

Theorem $\mathbf{1}_{\mathbf{1}}$. For any weight $w$ in $A_{1}^{\delta}(J)$, inequalities (4) are true with the sharp constants

$$
\begin{gathered}
C_{\min }(1, q, \delta)= \begin{cases}1, & q \in(-\infty, 0] \cup[1,+\infty), \\
\frac{\delta^{1-q}}{q+(1-q) \delta}, & q \in[0,1],\end{cases} \\
C_{\max }(1, q, \delta)= \begin{cases}+\infty, & q \in\left[\frac{\delta}{\delta-1},+\infty\right), \\
\frac{\delta^{1-q}}{q+(1-q) \delta}, & q \in(-\infty, 0] \cup\left[1, \frac{\delta}{\delta-1}\right), \\
1, & q \in[0,1] .\end{cases}
\end{gathered}
$$

In spite of the fact that formulas (7)-(8) and (9)-(10) are limit cases of formulas (5)(6) (this is easy for $p \rightarrow \infty$, and not so easy, but also true for $p \rightarrow 1$ ) and the general method of the proof is the same for all $p$, we shall consider the case of $1<p<\infty$ first, and then show how it should be modified to get the proof for $p=\infty$ and for $p=1$.

So, assume that $1<p<\infty$. The Hölder inequality implies that if $w \in A_{p}^{\delta}(I)$, then the point $x=\left(\langle w\rangle_{I},\left\langle w^{1-p^{\prime}}\right\rangle_{I}\right)$ is in the domain

$$
\Omega_{\delta}(p) \stackrel{\text { def }}{=}\left\{x=\left(x_{1}, x_{2}\right): x_{2}>0,1 \leq x_{1} x_{2}^{p-1} \leq \delta\right\} .
$$

On $\Omega_{\delta}$ we define two functions:

$$
\begin{aligned}
& \mathbf{B}_{\max }(x ; p, q, \delta) \stackrel{\text { def }}{=} \sup _{w \in A_{p}^{\delta}(I)}\left\{\left\langle w^{q}\right\rangle_{I}:\langle w\rangle_{I}=x_{1},\left\langle w^{1-p^{\prime}}\right\rangle_{I}=x_{2}\right\}, \\
& \mathbf{B}_{\min }(x ; p, q, \delta) \stackrel{\text { def }}{=} \inf _{w \in A_{p}^{\delta}(I)}\left\{\left\langle w^{q}\right\rangle_{I}:\langle w\rangle_{I}=x_{1},\left\langle w^{1-p^{\prime}}\right\rangle_{I}=x_{2}\right\} .
\end{aligned}
$$

Since for every $x \in \Omega_{\delta}$ there exists a weight $w$ with $\langle w\rangle_{I}=x_{1}$ and $\left\langle w^{1-p^{\prime}}\right\rangle_{I}=x_{2}$ (for instance, such a weight will be constructed in the proof of Lemma 1), the functions $\mathbf{B}$ are well defined on the entire domain $\Omega_{\delta}$ (making some assertions concerning both functions $\mathbf{B}_{\max }$ and $\mathbf{B}_{\min }$, we shall omit the subscripts max and min).

It is obvious that the functions $\mathbf{B}$ do not depend on $I$. Indeed, for any two intervals $I_{1}$ and $I_{2}$, an affine mapping of one interval onto another puts the classes $A_{p}^{\delta}\left(I_{1}\right)$ and $A_{p}^{\delta}\left(I_{2}\right)$ in one-to-one correspondence, and such a change of the variable preserves the averages. Therefore, the supremum in (11) and the infimum in (12) do not depend on $I$. In spite of the fact that $\mathbf{B}$ depends on $\delta, p$, and $q$ essentially, sometimes we shall omit these parameters if it is clear that they are fixed. 
V. VASYUNIN

The functions defined in (11)-(12) are none other than the Bellman functions mentioned above. There are very few cases where the expression for a Bellman function can be written explicitly. Usually, instead of the "true" Bellman function, some simple analog of it is constructed in such a way that it still possesses certain key properties of the genuine Bellman function. Generally, these properties suffice if we do not aim at the sharp constants (see [2], 4]). The emphasis of this paper is on finding explicit expressions for the Bellman functions (11)-(12); see Theorem 2. We use the following notation:

$$
r^{ \pm}=r_{p}^{ \pm}(x ; \delta) \stackrel{\text { def }}{=} u_{p}^{ \pm}\left(\frac{1}{\delta} x_{1} x_{2}^{p-1}\right)
$$

Note that $s^{ \pm}=\left.r^{ \pm}(x ; \delta)\right|_{x_{1}=x_{2}^{1-p}}$.

Theorem 2. For $x_{1}=x_{2}^{1-p}$ we have

$$
\mathbf{B}_{\max }(x ; p, q, \delta)=\mathbf{B}_{\min }(x ; p, q, \delta)=x_{1}^{q} .
$$

Otherwise

$$
\begin{aligned}
& \mathbf{B}_{\max }(x ; p, q, \delta) \\
& = \begin{cases}x_{1}^{\gamma} x_{2}^{1-\gamma}\left(\frac{1-s^{+}}{1-r^{+}}\right)^{\gamma} \frac{1-\gamma r^{+}}{1-\gamma s^{+}}, & \gamma \in\left(0, \frac{1}{p}\right] \cup\left[1, \frac{1}{s^{+}}\right), \\
x_{1}^{\gamma} x_{2}^{1-\gamma}\left(\frac{1-s^{-}}{1-r^{-}}\right)^{\gamma} \frac{1-\gamma r^{-}}{1-\gamma s^{-}}, & \gamma \in\left(\frac{1}{s^{-}}, 0\right] \cup\left[\frac{1}{p}, 1\right], \\
+\infty, & \gamma \in\left(-\infty, \frac{1}{s^{-}}\right] \cup\left[\frac{1}{s^{+}},+\infty\right),\end{cases}
\end{aligned}
$$

and

$$
\begin{aligned}
& \mathbf{B}_{\min }(x ; p, q, \delta) \\
& = \begin{cases}x_{1}^{\gamma} x_{2}^{1-\gamma}\left(\frac{1-s^{-}}{1-r^{-}}\right)^{\gamma} \frac{1-\gamma r^{-}}{1-\gamma s^{-}}, & \gamma \in\left(0, \frac{1}{p}\right] \cup[1,+\infty), \\
x_{1}^{\gamma} x_{2}^{1-\gamma}\left(\frac{1-s^{+}}{1-r^{+}}\right)^{\gamma} \frac{1-\gamma r^{+}}{1-\gamma s^{+}}, & \gamma \in(-\infty, 0] \cup\left[\frac{1}{p}, 1\right] .\end{cases}
\end{aligned}
$$

Theorem 1 is an immediate consequence of Theorem 2 . To deduce Theorem 1 from Theorem 2, we must merely calculate the supremum of $x_{1}^{-q} \mathbf{B}_{\max }(x ; p, q, \delta)$ and the infimum of $x_{1}^{-q} \mathbf{B}_{\min }(x ; p, q, \delta)$ when $x$ runs over $\Omega_{\delta}$.

Before we do this, one more remark concerning notation is in order. In Theorems 1 and 2 , the same five critical points are written in different form, in terms of $q$ in Theorem 1 and in terms of $\gamma=\frac{p q-q-1}{p}$ in Theorem 2:

$$
\begin{array}{cccccc}
q: & \frac{p-s^{-}}{s^{-}(p-1)} & -\frac{1}{p-1} & 0 & 1 & \frac{p-s^{+}}{s^{+}(p-1)} \\
\gamma: & \frac{1}{s^{-}} & 0 & \frac{1}{p} & 1 & \frac{1}{s^{+}} .
\end{array}
$$

Now, using the definitions of $r$ and $s$ (for both indices \pm ), i.e.,

$$
\frac{1}{\delta} x_{1} x_{2}^{p-1}=(1-r)\left(1-\frac{r}{p}\right)^{-p}, \quad \frac{1}{\delta}=(1-s)\left(1-\frac{s}{p}\right)^{-p}
$$

we arrive at the relation

$$
x_{1} x_{2}^{p-1}=\delta \frac{1-r}{(1-r / p)^{p}}=\frac{1-r}{1-s}\left(\frac{p-s}{p-r}\right)^{p} .
$$

Thus, we can rewrite (13) or (14) differently:

$$
\mathbf{B}=x_{1}^{\gamma} x_{2}^{1-\gamma}\left(\frac{1-s}{1-r}\right)^{\gamma} \frac{1-\gamma r}{1-\gamma s}=x_{1}^{q}\left(\frac{1-s}{1-r}\right)^{q}\left(\frac{p-r}{p-s}\right)^{q-1} \frac{p-(p q-q+1) r}{p-(p q-q+1) s} .
$$


To get the constants $C_{\min }$ and $C_{\max }$, we must investigate

$$
g \stackrel{\text { def }}{=} x_{1}^{-q} \mathbf{B}=\left(\frac{1-s}{1-r}\right)^{q}\left(\frac{p-r}{p-s}\right)^{q-1} \frac{p-(p q-q+1) r}{p-(p q-q+1) s}
$$

as functions of the variable $r$. Since

$$
\frac{\partial}{\partial r} \log g=\frac{g^{\prime}}{g}=-\frac{q(q-1)(p-1)^{2} r}{p(1-r)(1-\gamma r)(p-r)},
$$

the sign of $g^{\prime}$ coincides with the sign of $q(1-q) r$. Indeed, we always have $r<1<p$. Next, $\gamma r<1$ for $r \in\left[s^{-}, 0\right]$ and $1 / s^{-}<\gamma<+\infty$ as well as for $r \in\left[0, s^{+}\right]$and $-\infty<\gamma<1 / s^{+}$. Therefore, the function $g(r)$ is monotone increasing on $\left(s^{-}, 0\right]$ and monotone decreasing on $\left[0, s^{+}\right)$for $q \notin[0,1]$, and it has the opposite behavior for $q \in(0,1)$. We shall mark the function $g$ by the upper index + or - depending on the choice of the sign for $r$ and by the lower index max or min depending on the corresponding index of the function $\mathbf{B}$. Hence, for $q<-\frac{1}{p-1}$ the function $g^{+}=g_{\min }$ takes its minimum value at $r^{+}=s^{+}$, and $g^{-}=g_{\text {max }}$ takes its maximum value at $r^{-}=0$, i.e.,

$$
x_{1}^{q} \leq \mathbf{B}_{\min }(x ; p, q, \delta) \leq \mathbf{B}_{\max }(x ; p, q, \delta) \leq \frac{\left(1-s^{-}\right)^{q}}{1-\gamma s^{-}}\left(1-\frac{s^{-}}{p}\right)^{1-q} x_{1}^{q} .
$$

For $q>1$ and for $-\frac{1}{p-1}<q<0$, the function $g^{-}=g_{\min }$ takes its minimum value at $r^{-}=s^{-}$, and $g^{+}=g_{\max }$ takes its maximum value at $r^{+}=0$, i.e.,

$$
x_{1}^{q} \leq \mathbf{B}_{\min }(x ; p, q, \delta) \leq \mathbf{B}_{\max }(x ; p, q, \delta) \leq \frac{\left(1-s^{+}\right)^{q}}{1-\gamma s^{+}}\left(1-\frac{s^{+}}{p}\right)^{1-q} x_{1}^{q} .
$$

For $0<q<1$, the function $g^{+}=g_{\min }$ takes its minimum value at $r^{+}=0$, and $g^{-}=g_{\max }$ takes its maximum value at $r^{-}=s^{-}$, i.e.,

$$
\frac{\left(1-s^{+}\right)^{q}}{1-\gamma s^{+}}\left(1-\frac{s^{+}}{p}\right)^{1-q} x_{1}^{q} \leq \mathbf{B}_{\min }(x ; p, q, \delta) \leq \mathbf{B}_{\max }(x ; p, q, \delta) \leq x_{1}^{q} .
$$

To get the constants $C_{\min }, C_{\max }$ in the form (5)-(6), it suffices to observe that

$$
(1-s)^{q}\left(1-\frac{s}{p}\right)^{1-q}=\delta^{\frac{1-q}{p}}(1-s)^{\gamma} .
$$

We note that the functions $\mathbf{B}$ depend continuously on the parameter $q$. Moreover, at the endpoints of intervals with different analytical expressions these functions coincide, namely

$$
\begin{aligned}
\mathbf{B}\left(x ; p, 1-p^{\prime}, \delta\right) & =x_{2}, \\
\mathbf{B}(x ; p, 0, \delta) & =1, \\
\mathbf{B}(x ; p, 1, \delta) & =x_{1} .
\end{aligned}
$$

Corollary to Theorem 1. For the weights $w \in A_{p}^{\delta}(J)$, the Muckenhoupt condition $\left(A_{\mathbf{p}}\right)$ is fulfilled for every $\mathbf{p}>\frac{p\left(1-s^{-}\right)}{p-s^{-}}$with the sharp constant

$$
\sup _{I \subset J}\langle w\rangle_{I}\left\langle w^{1-\mathbf{p}^{\prime}}\right\rangle_{I}^{\mathbf{p}-1}=\frac{(1-s / p)^{\mathbf{p}}}{(1-s)\left(1-\frac{\mathbf{p}-p}{p(\mathbf{p}-1)} s\right)^{\mathbf{p}-1}},
$$

where $s=s^{-}$for $\mathbf{p} \leq p$ and $s=s^{+}$for $\mathbf{p} \geq p$.

To obtain this estimate, it suffices to apply Theorem 1 with $q=1-\mathbf{p}^{\prime}$. 
V. VASYUNIN

Outline of the proof of Theorem 2. Immediately after the introductory remarks, a rigorous proof of formulas (13)-(14) will be presented. However, it conceals a part of the truth because it requires the prior knowledge of the above explicit formulas for the Bellman functions. The procedure leading to this "guess" is described in Appendix 1. In a standard way the matter is reduced to the Bellman equation. By the specific character of the problem, this equation turns into the ordinary differential equation (45), which, moreover, admits explicit solution. However, the deduction of formulas (13)-(14) in Appendix 1 is not a proof, because it is based on a series of voluntary assumptions (e.g., the Bellman function is assumed to be sufficiently smooth, which is not clear a priori). For this reason, the heuristic arguments in Appendix 1 must be supplemented with a rigorous proof, to which we pass now.

We denote by $B_{\max }(x ; p, q, \delta)$ and $B_{\min }(x ; p, q, \delta)$ the functions on the right-hand side of (13) and (14), respectively, i.e., we put

$$
\begin{gathered}
B_{\max }(x ; p, q, \delta) \stackrel{\text { def }}{=} \begin{cases}x_{1}^{\gamma} x_{2}^{1-\gamma}\left(\frac{1-s^{+}}{1-r^{+}}\right)^{\gamma} \frac{1-\gamma r^{+}}{1-\gamma s^{+}}, & \gamma \in\left(0, \frac{1}{p}\right] \cup\left[1, \frac{1}{s^{+}}\right), \\
x_{1}^{\gamma} x_{2}^{1-\gamma}\left(\frac{1-s^{-}}{1-r^{-}}\right)^{\gamma} \frac{1-\gamma r^{-}}{1-\gamma s^{-}}, & \gamma \in\left(\frac{1}{s^{-}}, 0\right] \cup\left[\frac{1}{p}, 1\right], \\
+\infty, & \gamma \in\left(-\infty, \frac{1}{s^{-}}\right] \cup\left[\frac{1}{s^{+}},+\infty\right),\end{cases} \\
B_{\min }(x ; p, q, \delta) \stackrel{\text { def }}{=} \begin{cases}x_{1}^{\gamma} x_{2}^{1-\gamma}\left(\frac{1-s^{-}}{1-r^{-}}\right)^{\gamma} \frac{1-\gamma r^{-}}{1-\gamma s^{-}}, & \gamma \in\left(0, \frac{1}{p}\right] \cup[1, \infty), \\
x_{1}^{\gamma} x_{2}^{1-\gamma}\left(\frac{1-s^{+}}{1-r^{+}}\right)^{\gamma} \frac{1-\gamma r^{+}}{1-\gamma s^{+}}, & \gamma \in(-\infty, 0] \cup\left[\frac{1}{p}, 1\right] .\end{cases}
\end{gathered}
$$

The identities $\mathbf{B}_{\max }=B_{\max }$ and $\mathbf{B}_{\min }=B_{\min }$ will be obtained from the inequalities " $\leq$ " and " $\geq "$ ". We shall start with verifying two easier estimates among the four.

Lemma 1. The inequalities

$$
\mathbf{B}_{\max }(x ; p, q, \delta) \geq B_{\max }(x ; p, q, \delta), \quad \mathbf{B}_{\min }(x ; p, q, \delta) \leq B_{\min }(x ; p, q, \delta)
$$

are true for every $x \in \Omega_{\delta}$ and every $q \in \mathbb{R}, 1<p<\infty$.

These inequalities will be proved by exhibiting an explicit extremal function (in Appendix 2 it will be explained how to find it). The reverse inequalities resist direct verification, and an approximation procedure will be used; namely, we shall prove the following lemma.

Lemma 2. The inequalities

$$
\mathbf{B}_{\max }(x ; p, q, \delta) \leq B_{\max }(x ; p, q, \varepsilon), \quad \mathbf{B}_{\min }(x ; p, q, \delta) \geq B_{\min }(x ; p, q, \varepsilon)
$$

are true for every $x \in \Omega_{\delta}$, every $q \in \mathbb{R}, 1<p<\infty$, and every $\varepsilon>\delta$.

Passage to the limit as $\varepsilon \rightarrow \delta$ is possible here, which yields the second pair of inequalities. Indeed, $B_{\min }$ is a continuous function of the parameter $\delta$. As to $B_{\max }$, we consider the finite and the infinite values of this function separately. If $\gamma \geq 1 / s^{+}(\delta)$ (or $\gamma \leq 1 / s^{-}(\delta)$ ), then $\gamma \geq 1 / s^{+}(\varepsilon)$ (respectively, $\gamma \leq 1 / s^{-}(\varepsilon)$ ) for all $\varepsilon>\delta$. Therefore, $\mathbf{B}_{\max }(x ; p, q, \delta)=B_{\max }(x ; p, q, \varepsilon)=+\infty$, and there is nothing to prove. If $1 / s^{-}(\delta)<\gamma<$ $1 / s^{+}(\delta)$, then $1 / s^{-}(\varepsilon)<\gamma<1 / s^{+}(\varepsilon)$ for $\varepsilon$ sufficiently close to $\delta$ (because the functions $\delta \mapsto s^{ \pm}(\delta)$ are continuous and $\left|s^{ \pm}(\delta)\right|$ monotonically increases with $\delta$ ). Therefore, if we restrict ourselves to $\varepsilon$ sufficiently close to $\delta$, then $B_{\max }$ becomes finite and continuous in the parameter $\delta$, and we can pass to the limit as $\varepsilon \rightarrow \delta$.

We start the proof of Lemma 2 with verifying that the function $B_{\max }$ is concave and the function $B_{\min }$ is convex. 
Lemma 3. Let $x^{ \pm}$be two arbitrary points in $\Omega_{\delta}$. If the entire interval $\left[x^{-}, x^{+}\right]$between these two points is in $\Omega_{\delta}$, then the inequalities

$$
\begin{gathered}
B_{\max }\left(\alpha^{-} x^{-}+\alpha^{+} x^{+}\right) \geq \alpha^{-} B_{\max }\left(x^{-}\right)+\alpha^{+} B_{\max }\left(x^{+}\right), \\
B_{\min }\left(\alpha^{-} x^{-}+\alpha^{+} x^{+}\right) \leq \alpha^{-} B_{\min }\left(x^{-}\right)+\alpha^{+} B_{\min }\left(x^{+}\right)
\end{gathered}
$$

are true for an arbitrary pair of nonnegative numbers $\alpha^{ \pm}$such that $\alpha^{-}+\alpha^{+}=1$.

In the proof of Lemma 2 we shall encounter the following situation. Given a weight $w \in A_{p}^{\delta}(J)$, we split an arbitrary interval $I, I \subset J$, into two parts $I=I^{-} \cup I^{+}$and get two points in $\Omega_{\delta}, x^{ \pm}=\left(\langle w\rangle_{I^{ \pm}},\left\langle w^{1-p^{\prime}}\right\rangle_{I^{ \pm}}\right)$, to which we want to apply Lemma 3 . To do this, we need the whole interval $\left[x^{-}, x^{+}\right]$be inside $\Omega_{\delta}$, but, generally speaking, this is not so. Then we enlarge the domain a bit and try to split the interval $I$ in such a way that the interval $\left[x^{-}, x^{+}\right]$would be inside $\Omega_{\varepsilon}, \varepsilon>\delta$. The existence of the required splitting is ensured by the following lemma.

Lemma 4. If a parameter $\delta>1$ is given, then for an arbitrary $\varepsilon>\delta$ and an arbitrary weight $w \in A_{p}^{\delta}(I)$ there exists a splitting $I=I^{-} \cup I^{+},\left|I^{ \pm}\right|=\alpha^{ \pm}|I|$, such that the entire interval with the endpoints $x^{ \pm}=\left(\langle w\rangle_{I^{ \pm}},\left\langle w^{1-p^{\prime}}\right\rangle_{I^{ \pm}}\right)$is in $\Omega_{\varepsilon}$. Moreover, the splitting parameters $\alpha^{ \pm}$can be chosen bounded away from 0 and 1 uniformly with respect to $w$ and, therefore, with respect to I as well.

Now we begin to realize the program outlined above.

Proof of Lemma 1. Since $x_{2}=x_{1}^{1-p^{\prime}}$ if and only if $w=x_{1}=$ const, it is clear that $\mathbf{B}\left(x_{1}, x_{1}^{1-p^{\prime}} ; p, q, \delta\right)=B\left(x_{1}, x_{1}^{1-p^{\prime}} ; p, q, \delta\right)=x_{1}^{q}$ for all $x_{1}>0, q \in \mathbb{R}$, and $p \in(1,+\infty)$. So we need to consider $\delta>1$ and the points $x$ with $x_{1} x_{2}^{p-1}>1$.

Fix an arbitrary point $x$ in $\Omega_{\delta}$ with $x_{1} x_{2}^{p-1}>1$. Let $a \in(0,1]$ and $J=[0,1]$. We put

$$
w_{c, a, \nu}(t)= \begin{cases}c a^{\nu} t^{-\nu} & \text { if } 0 \leq t \leq a, \\ c & \text { if } a \leq t \leq 1 .\end{cases}
$$

Direct calculation shows that

$$
\left\langle w_{c, a, \nu}^{\theta}\right\rangle_{J}= \begin{cases}c^{\theta} \frac{1-(1-a) \theta \nu}{1-\theta \nu} & \text { if } \theta \nu<1, \\ \infty & \text { if } \theta \nu \geq 1 .\end{cases}
$$

So, if we would like to get a function with a finite $A_{p^{-}}$"norm", we must restrict ourselves to $\nu \in(1-p, 1)$. For such $\nu$ the $A_{p^{-}}$"norm" of $w_{c, a, \nu}$ is finite indeed (the calculation of it is moved to Appendix 3). To get an extremal function for $\mathbf{B}$, we take

$$
\nu=\frac{(p-1) s}{p-s}, \quad a=\frac{(s-r) p}{(p-r) s}, \quad c=\frac{(p-r)(1-s)}{(p-s)(1-r)} x_{1}
$$

with an appropriate choice of the signs for $s$ and $r$; namely, in accordance with (13)-(14),

$$
\begin{array}{ll}
s=s_{p}^{+}(\delta), r=r_{p}^{+}(x ; \delta) & \begin{cases}\text { for } \mathbf{B}_{\max } & \text { if } q \in\left[1-p^{\prime}, 0\right] \cup[1,+\infty), \\
f \circ \mathbf{B}_{\min } & \text { if } q \in\left(-\infty, 1-p^{\prime}\right] \cup[0,1],\end{cases} \\
s=s_{p}^{-}(\delta), r=r_{p}^{-}(x ; \delta) & \begin{cases}\text { for } \mathbf{B}_{\min } & \text { if } q \in\left[1-p^{\prime}, 0\right] \cup[1,+\infty), \\
f \circ \mathbf{B}_{\max } & \text { if } q \in\left(-\infty, 1-p^{\prime}\right] \cup[0,1] .\end{cases}
\end{array}
$$

Recall that $s^{ \pm}=u^{ \pm}(p, 1 / \delta)$, i.e., $\delta(1-s)(1-s / p)^{-p}=1$, and $r^{ \pm}=u^{ \pm}\left(p, \frac{1}{\delta} x_{1} x_{2}^{p-1}\right)$, i.e., $\delta(1-r)(1-r / p)^{-p}=x_{1} x_{2}^{p-1}$. Since the condition $\delta>1$ has been assumed, we are sure that $s^{ \pm} \neq 0$ and $a$ is well defined. Moreover, $a \in(0,1]$. Indeed, on the one hand, $r / s<1$ (since we have assumed that $x_{1} x_{2}^{p-1}>1$ ), i.e., $\frac{s-r}{s}>0$, and $p>1>r$, so that 
$a>0$. On the other hand, $r / s \geq 0$ and $p>1>s$, whence $1-a=\frac{(p-s) r}{(p-r) s} \geq 0$, i.e., $a \leq 1$. As to the parameter $\nu$, it is easily seen that the condition $s<1$ implies that $\nu$ is inside the admissible interval $1-p<\nu<1$.

In Appendix 3 the $A_{p}$-"norm" of the function $w_{c, a, \nu}$ will be calculated: it is equal to $\frac{1}{1-\nu}\left(1+\frac{\nu}{p-1}\right)^{1-p}$. Since this is equal to $\delta$ for the value of $\nu$ chosen above, we have $w_{c, a, \nu} \in A_{p}^{\delta}(I)$.

Now, using (20), we check that the weight $w_{c, a, \nu}$ represents the point $x=\left(x_{1}, x_{2}\right)$ in $\Omega_{\delta}$. First, we have

$$
\left\langle w_{c, a, \nu}\right\rangle_{J}=c \frac{1-(1-a) \nu}{1-\nu}=x_{1} .
$$

To calculate the second coordinate, we rewrite the expression for $c$ by using (15):

$$
c=\left(\frac{p-s}{p-r}\right)^{p-1} x_{2}^{1-p} .
$$

Then

$$
\left\langle w_{c, a, \nu}^{1-p^{\prime}}\right\rangle_{J}=c^{1-p^{\prime}} \cdot \frac{1-(1-a)\left(1-p^{\prime}\right) \nu}{1-\left(1-p^{\prime}\right) \nu}=\frac{p-r}{p-s} \cdot x_{2} \cdot \frac{p-s}{p-r}=x_{2} .
$$

So, for $q \geq 1 / \nu^{+}=\frac{p-s^{+}}{(p-1) s^{+}}$and for $q \leq 1 / \nu^{-}=\frac{p-s^{-}}{(p-1) s^{-}}$we get $\mathbf{B}_{\max }(x ; p, q, \delta)=$ $B_{\max }(x ; p, q, \delta)=\infty$. By $(22)$ and $(15)$, for $q \nu<1$ we obtain

$$
\begin{aligned}
\left\langle w_{c, a, \nu}^{q}\right\rangle_{J} & =c^{q} \frac{1-(1-a) q \nu}{1-q \nu}=\left(\frac{p-s}{p-r}\right)^{q(p-1)} x_{2}^{q(1-p)} \frac{1-\frac{(p-s) r}{(p-r) s} q \frac{(p-1) s}{p-s}}{1-q \frac{(p-1) s}{p-s}} \\
& =\left(\frac{p-s}{p-r}\right)^{\gamma p-1} x_{2}^{1-\gamma p} \frac{1-\frac{(\gamma p-1) r}{p-r}}{1-\frac{(\gamma p-1) s}{p-s}}=x_{2}^{1-\gamma} x_{2}^{\gamma(1-p)}\left(\frac{p-s}{p-r}\right)^{\gamma p} \frac{1-\gamma r}{1-\gamma s} \\
& =x_{2}^{1-\gamma} x_{1}^{\gamma}\left(\frac{1-s}{1-r}\right)^{\gamma} \frac{1-\gamma r}{1-\gamma s}=B(x ; p, q, \delta),
\end{aligned}
$$

where we put $s=s^{+}$and $r=r^{+}$or $s=s^{-}$and $r=r^{-}$in accordance with (13) and (14). Now, the required inequalities follow from the definition of $\mathbf{B}$.

Proof of Lemma 3. To prove the lemma, we need to check that the matrix

$$
\left\{\frac{\partial^{2} B}{\partial x_{i} \partial x_{j}}\right\}_{i, j=1}^{2}
$$

is nonpositive for $B_{\max }$ and nonnegative for $B_{\min }$. Direct calculation yields

$$
\begin{gathered}
\frac{\partial r}{\partial x_{1}}=-\frac{(1-r)(p-r)}{(p-1) r x_{1}}, \quad \frac{\partial r}{\partial x_{2}}=-\frac{(1-r)(p-r)}{r x_{2}}, \\
\frac{\partial B}{\partial x_{1}}=\frac{\gamma q(1-r)}{x_{1}(1-\gamma r)} B, \quad \frac{\partial B}{\partial x_{2}}=\frac{(\gamma p-1)(\gamma-1)}{x_{2}(1-\gamma r)} B, \\
\frac{\partial^{2} B}{\partial x_{1}^{2}}=-\gamma q(q-1) \frac{x_{1}^{\gamma-2} x_{2}^{1-\gamma}}{r(1-r)^{\gamma-2}} \frac{(1-s)^{\gamma}}{1-\gamma s}, \\
\frac{\partial^{2} B}{\partial x_{1} \partial x_{2}}=-\gamma q(q-1)(p-1) \frac{x_{1}^{\gamma-1} x_{2}^{-\gamma}}{r(1-r)^{\gamma-1}} \frac{(1-s)^{\gamma}}{1-\gamma s}, \\
\frac{\partial^{2} B}{\partial x_{2}^{2}}=-\gamma q(q-1)(p-1)^{2} \frac{x_{1}^{\gamma} x_{2}^{-\gamma-1}}{r(1-r)^{\gamma}} \frac{(1-s)^{\gamma}}{1-\gamma s} .
\end{gathered}
$$


Therefore, the quadratic form of the matrix (23) is

$$
\sum_{i, j=1}^{2} \frac{\partial^{2} B}{\partial x_{i} \partial x_{j}} \Delta_{i} \Delta_{j}=-\frac{\gamma q(q-1) x_{1}^{\gamma-2} x_{2}^{1-\gamma}}{r(1-\gamma s)}\left(\frac{1-s}{1-r}\right)^{\gamma}\left(x_{2}(1-r) \Delta_{1}+(p-1) x_{1} \Delta_{2}\right)^{2}
$$

This expression has the required sign, because the value of $1-\gamma s$ is always positive and the sign of $r$ coincides with the sign of $\gamma q(q-1)$ for $B_{\max }$ and is opposite to the latter for $B_{\min }$.

Proof of Lemma 4. For any interval $I$, we describe a procedure of splitting it into two parts $I^{-}$and $I^{+},\left|I^{ \pm}\right|=\alpha^{ \pm}|I|$. As before, we put $x_{1}^{ \pm}=\langle w\rangle_{I^{ \pm}}$and $x_{2}^{ \pm}=\left\langle w^{1-p^{\prime}}\right\rangle_{I^{ \pm}}$. For all $\alpha^{+}$the interval with the endpoints $x^{ \pm}$(to be denoted by $\left[x^{-}, x^{+}\right]$) contains the point $x^{0}=\alpha^{-} x^{-}+\alpha^{+} x^{+}$whose coordinates are the corresponding averages over the entire interval $I: x_{1}^{0}=\langle w\rangle_{I}, x_{2}^{0}=\left\langle w^{1-p^{\prime}}\right\rangle_{I}$.

We start with taking $\alpha^{-}=\alpha^{+}=1 / 2$. If the entire segment $\left[x^{-}, x^{+}\right]$is in the domain $\Omega_{\varepsilon}$, we fix this splitting. In the remaining part of the proof we assume that this is not the case, i.e., there exists a point $x$ on this interval with $x_{1} x_{2}^{p-1}>\varepsilon$. Since any straight line intersects the graph of a power function at two points at most, we can choose $\xi$ to be the endpoint of our segment such that all points $x$ with $x_{1} x_{2}^{p-1}>\varepsilon$ are between $x^{0}$ and $\xi$. We define a function $\rho\left(\alpha^{+}\right)$as follows: given $\alpha^{+}$, we put $\rho\left(\alpha^{+}\right)$to be the maximum value of $x_{1} x_{2}^{p-1}$, where $x$ runs over the segment between $x^{0}$ and $\xi$. By our assumption, $\rho(1 / 2)>\varepsilon$. We begin to change $\alpha^{+}$so as to make $\xi$ go towards $x^{0}$, i.e., we increase $\alpha^{+}$ if $\xi=x^{+}$, and reduce it if $\xi=x^{-}$. We stop at the moment when the relation $\rho\left(\alpha^{+}\right)=\varepsilon$ occurs for the first time. This finishes the description of the splitting procedure. Now, we must check that the moment indicated above does exist and, moreover, the corresponding value of $\alpha^{+}$is bounded away from 0 and 1 uniformly with respect to $w$ and $I$.

Since $x_{1}^{0}\left(x_{2}^{0}\right)^{p-1} \leq \delta$, we have $x_{1} x_{2}^{p-1}<\varepsilon$ on the entire segment between $x^{0}$ and $\xi$ if $\xi$ is sufficiently close to $x^{0}$, i.e., $\rho\left(\alpha^{+}\right)<\varepsilon$ for the corresponding $\alpha^{+}$. The function $\rho$ is continuous, which implies the existence of $\alpha^{+}$such that $\rho\left(\alpha^{+}\right)=\varepsilon$.

Thus, we have proved that the "stopping time" described above exists, and we must check that the corresponding $\alpha^{+}$is not too close to 0 and 1 . If $\xi=x^{+}$, then $\alpha^{+}>1 / 2$ and $\xi_{1}-x_{1}^{0}=x_{1}^{+}-x_{1}^{0}=\alpha^{-}\left(x_{1}^{+}-x_{1}^{-}\right)$. Symmetrically, if $\xi=x^{-}$, then $\alpha^{-}>1 / 2$ and $\xi_{1}-x_{1}^{0}=x_{1}^{-}-x_{1}^{0}=\alpha^{+}\left(x_{1}^{-}-x_{1}^{+}\right)$. Thus, $\left|\xi_{1}-x_{1}^{0}\right|=\min \left\{\alpha^{ \pm}\right\}\left|x_{1}^{-}-x_{1}^{+}\right|$.

For the "stopping" value of $\alpha^{+}$the straight line that passes through the points $x^{ \pm}$and $x^{0}$ is tangent to the graph $x_{1} x_{2}^{p-1}=\varepsilon$ and touches it at some point, say $\tau$. The equation of the line tangent to the graph $x_{1} x_{2}^{p-1}=$ const at the point $\tau$ is $\tau_{2} x_{1}+(p-1) \tau_{1} x_{2}=p \tau_{1} \tau_{2}$. This line intersects the graph $x_{1} x_{2}^{p-1}=\delta$ at the points

$$
x(\delta)^{ \pm}=\left(\tau_{1}\left[1-u_{1-p}^{ \pm}\left(\frac{\delta}{\varepsilon}\right)\right], \tau_{2}\left[1+\frac{1}{p-1} u_{1-p}^{ \pm}\left(\frac{\delta}{\varepsilon}\right)\right]\right)
$$

and the graph $x_{1} x_{2}^{p-1}=1$ at the points

$$
x(1)^{ \pm}=\left(\tau_{1}\left[1-u_{1-p}^{ \pm}\left(\frac{1}{\varepsilon}\right)\right], \tau_{2}\left[1+\frac{1}{p-1} u_{1-p}^{ \pm}\left(\frac{1}{\varepsilon}\right)\right]\right) .
$$

So, we have got the following embedded segments of our line: $\left[x(\delta)^{-}, x(\delta)^{+}\right] \subset\left[x^{0}, \xi\right] \subset$ $\left[x^{-}, x^{+}\right] \subset\left[x(1)^{-}, x(1)^{+}\right]$. Therefore,

$$
\left|x(\delta)_{1}^{+}-x(\delta)_{1}^{-}\right| \leq\left|x_{1}^{0}-\xi_{1}\right|=\min \left\{\alpha^{ \pm}\right\}\left|x_{1}^{+}-x_{1}^{-}\right| \leq \min \left\{\alpha^{ \pm}\right\}\left|x(1)_{1}^{+}-x(1)_{1}^{-}\right|
$$

and

$$
\min \left\{\alpha^{ \pm}\right\} \geq \frac{\left|x(\delta)_{1}^{+}-x(\delta)_{1}^{-}\right|}{\left|x(1)_{1}^{+}-x(1)_{1}^{-}\right|}=\frac{\left|u_{1-p}^{+}(\delta / \varepsilon)-u_{1-p}^{-}(\delta / \varepsilon)\right|}{\left|u_{1-p}^{+}(1 / \varepsilon)-u_{1-p}^{-}(1 / \varepsilon)\right|} .
$$


We see that this estimate depends on $p, \delta$, and $\varepsilon$ only, but not on the choice of $w$ and I.

Remark. In the proof, we have used the functions $u_{p}^{ \pm}$for negative $p$ without formal definition. But it is easy to check that the mapping $t \mapsto(1-t)(1-t / p)^{-p}$ has two inverses defined on $[0,1]$ not only for $p>1$, but also for $p<0$. For negative $p$ the function $u_{p}^{+}$maps $[0,1]$ onto $[0,1]$, but $u_{p}^{-}$maps $[0,1]$ onto $[p, 0]$. There exists a simple rational relation between the functions $u_{p}$ and $u_{1-p}$ :

$$
\left(1-\frac{u_{p}}{p}\right)\left(1-\frac{u_{1-p}}{1-p}\right)=1 \text {. }
$$

Indeed, if we define the function $u_{1-p}$ with $p>1$ by (25), then

$$
\begin{gathered}
\left(1-u_{1-p}\right)\left(1-\frac{u_{1-p}}{1-p}\right)^{p-1}=\left(1-\frac{(p-1) u_{p}}{p-u_{p}}\right)\left(1+\frac{u_{p}}{p-u_{p}}\right)^{p-1} \\
=\frac{p\left(1-u_{p}\right)}{p-u_{p}}\left(\frac{p}{p-u_{p}}\right)^{p-1}=\left(1-u_{p}\right)\left(1-\frac{u_{p}}{p}\right)^{-p} .
\end{gathered}
$$

Since in (25) $u_{p}$ and $u_{1-p}$ have the same sign, the definition of $u_{p}^{ \pm}$for negative $p$ as the inverses to the mapping $t \mapsto(1-t)(1-t / p)^{-p}$ is equivalent to the definition by formula (25).

Now, we are ready to prove the main lemma.

Proof of Lemma 2. The statement of the lemma means that the inequalities

$$
B_{\min }(x ; p, q, \varepsilon) \leq\left\langle w^{q}\right\rangle_{I} \leq B_{\max }(x ; p, q, \varepsilon)
$$

are true for an arbitrary weight $w \in A_{p}^{\delta}(I)$ and an arbitrary $\varepsilon>\delta$. Here $x=\left(x_{1}, x_{2}\right)=$ $\left(\langle w\rangle_{I},\left\langle w^{1-p^{\prime}}\right\rangle_{I}\right)$. It suffices to prove (26) for step functions $w$ because an arbitrary weight $w$ can be approximated by a sequence of step functions $w_{n}$ so that all averages converge: $\left\langle w_{n}^{q}\right\rangle \rightarrow\left\langle w^{q}\right\rangle$ and $\left(\left\langle w_{n}\right\rangle_{I},\left\langle w_{n}^{1-p^{\prime}}\right\rangle_{I}\right) \rightarrow\left(\langle w\rangle_{I},\left\langle w^{1-p^{\prime}}\right\rangle_{I}\right)$, and we can pass to the limit in (26) because $B_{\min }$ is continuous in $x$, and so is $B_{\max }$ if it is finite, but for infinite $B_{\max }$ the inequality clearly holds.

So, we fix an interval $I$, a step function $w \in A_{p}^{\delta}(I)$, and a number $\varepsilon>\delta$. Starting with the interval $I=I^{0,0}$, we construct a chain of intervals $I^{n, m}$ in accordance with the rule in Lemma 4 . For the intervals in the $n$th generation, we use the notation $I^{n, m}$ : $I^{n, 2 k}=\left(I^{n-1, k}\right)^{-}$and $I^{n, 2 k+1}=\left(I^{n-1, k}\right)^{+}$, and so the second index runs from 0 to $2^{n}-1$. The corresponding mean values will be labeled by the same pair of indices, and $\alpha^{n, m}=\left|I^{n, m}\right| /|I|$. By Lemma 3, we can write the following chain of inequalities:

$$
\begin{aligned}
B_{\max }\left(x^{0,0} ; p, q, \varepsilon\right) & \geq \alpha^{1,0} B_{\max }\left(x^{1,0} ; p, q, \varepsilon\right)+\alpha^{1,1} B_{\max }\left(x^{1,1} ; p, q, \varepsilon\right) \\
& \geq \sum_{m=0}^{2^{n}-1} \alpha^{n, m} B_{\max }\left(x^{n, m} ; p, q, \varepsilon\right) .
\end{aligned}
$$

For $B_{\min }$ we get a similar chain of reverse inequalities. The latter sum tends to $\left\langle w^{q}\right\rangle_{I}$ as $n \rightarrow \infty$. Indeed, for a fixed step function $w \in A_{p}^{\delta}(J)$, the set $\left\{x=\left(\langle w\rangle_{I},\left\langle w^{1-p^{\prime}}\right\rangle_{I}\right): I \subset J\right\}$ is a compact subset of $\Omega_{\delta}$, and, therefore, the continuous function $B$ is bounded on this subset, say, $B \leq M$, i.e., $B\left(x^{n, m} ; p, q, \varepsilon\right) \leq M$ (we exclude the case of $B=B_{\max }=\infty$, when we have nothing to prove). So, if $N$ is the number of discontinuity points for the step function $w$, then the number of the intervals $I^{n, m}$ where $w$ is not a constant is at most $N$. On all other intervals $w$ is a constant, so that $B\left(x^{n, m} ; p, q, \varepsilon\right)=\left(x_{1}^{n, m}\right)^{q}=w^{q}$, i.e., the corresponding summand is $\frac{1}{\mid I} \int_{I^{n, m}} w(t)^{q} d t$, and the entire sum differs from $\left\langle w^{q}\right\rangle_{I}$ at most by $N M \max _{m} \alpha^{m, n}$. The latter quantity tends to zero, because by Lemma 4 
the values $\alpha^{ \pm}$are bounded away from 0 and 1, and the maximum length of the $n$th generation intervals tends to 0 as $n \rightarrow \infty$.

Now we consider the case of $p=\infty$. By the Jensen inequality, we see that $e^{\langle\log w\rangle} \leq$ $\langle w\rangle$. Therefore, for arbitrary $w \in A_{\infty}^{\delta}(I)$ the point $x=\left(x_{1}, x_{2}\right)=\left(\langle w\rangle_{I},\langle\log w\rangle_{I}\right)$ is in the domain

$$
\Omega_{\delta}(\infty) \stackrel{\text { def }}{=}\left\{\left(x_{1}, x_{2}\right): x_{2} \in \mathbb{R}, 1 \leq x_{1} e^{-x_{2}} \leq \delta\right\} .
$$

On $\Omega_{\delta}(\infty)$ we define the following functions:

$$
\begin{aligned}
& \mathbf{B}_{\max }(x ; q, \delta) \\
& \quad=\mathbf{B}_{\max }(x ; \infty, q, \delta) \stackrel{\text { def }}{=} \sup _{w \in A_{\infty}^{\delta}(I)}\left\{\left\langle w^{q}\right\rangle_{I}:\langle w\rangle_{I}=x_{1},\langle\log w\rangle_{I}=x_{2}\right\}, \\
& \mathbf{B}_{\min }(x ; q, \delta) \\
& \quad=\mathbf{B}_{\min }(x ; \infty, q, \delta) \stackrel{\text { def }}{=} \inf _{w \in A_{\infty}^{\delta}(I)}\left\{\left\langle w^{q}\right\rangle_{I}:\langle w\rangle_{I}=x_{1},\langle\log w\rangle_{I}=x_{2}\right\} .
\end{aligned}
$$

To keep continuity as $p \rightarrow \infty$, for finite $p$ we should have chosen

$$
x_{2}=-(p-1) \log \left\langle w^{\frac{1}{1-p}}\right\rangle_{I}
$$

rather than $x_{2}=\left\langle w^{\frac{1}{1-p}}\right\rangle_{I}$, because

$$
-(p-1) \log \left\langle w^{\frac{1}{1-p}}\right\rangle_{I} \approx-(p-1) \log \left(1-\frac{1}{p-1}\langle\log w\rangle_{I}\right) \rightarrow\langle\log w\rangle_{I}
$$

For this choice of $x_{2}$ the domain $\Omega_{\delta}(p)$ becomes the same for all $p$. Nevertheless, the choice of $x_{2}$ was made with the purpose to obtain a simple concavity/convexity condition for the functions $\mathbf{B}$ (see Lemma 3) rather than to get continuity as $p \rightarrow \infty$. So, comparison of the expressions for $\mathbf{B}(x ; p, q, \delta)$ and $\mathbf{B}(x ; \infty, q, \delta)$ requires the substitute $x_{2}^{p-1} \rightarrow e^{-x_{2}}$.

In the following theorem the explicit expression for the functions $\mathbf{B}(x ; \infty, q, \delta)$ will be given. In the statement and subsequently, we use the following notation: $r^{ \pm}=$ $r_{\infty}^{ \pm}(x ; \delta) \stackrel{\text { def }}{=} u_{\infty}^{ \pm}\left(\frac{1}{\delta} x_{1} e^{-x_{2}}\right)$ and $s^{ \pm}=s_{\infty}^{ \pm}(\delta) \stackrel{\text { def }}{=} u_{\infty}^{ \pm}(1 / \delta)$.

Theorem $\mathbf{2}_{\infty}$. For $x_{1}=e^{x_{2}}$, we have

$$
\mathbf{B}_{\max }(x ; q, \delta)=\mathbf{B}_{\min }(x ; q, \delta)=x_{1}^{q} .
$$

Otherwise

and

$$
\mathbf{B}_{\max }(x ; q, \delta)= \begin{cases}+\infty & \text { if } q \geq \frac{1}{s^{+}} \\ x_{1}^{q}\left(\frac{1-s^{+}}{1-r^{+}}\right)^{q} \frac{1-q r^{+}}{1-q s^{+}} & \text {if } 1 \leq q<\frac{1}{s^{+}} \\ x_{1}^{q}\left(\frac{1-s^{-}}{1-r^{-}}\right)^{q} \frac{1-q r^{-}}{1-q s^{-}} & \text {if } \frac{1}{s^{-}}<q \leq 1, \\ +\infty & \text { if } q \leq \frac{1}{s^{-}}\end{cases}
$$

$$
\mathbf{B}_{\min }(x ; q, \delta)= \begin{cases}x_{1}^{q}\left(\frac{1-s^{-}}{1-r^{-}}\right)^{q} \frac{1-q r^{-}}{1-q s^{-}} & \text {if } q \geq 1, \\ x_{1}^{q}\left(\frac{1-s^{+}}{1-r^{+}}\right)^{q} \frac{1-q r^{+}}{1-q s^{+}} & \text {if } q \leq 1 .\end{cases}
$$

Now, Theorem $1_{\infty}$ is an easy consequence. Indeed, for $x \in \Omega_{\delta}$ the parameter $r^{+}$runs over the interval $\left[0, s^{+}\right]$and $r^{-}$runs over the interval $\left[s^{-}, 0\right]$. If $q>1$, then the function $r \mapsto \frac{1-r q}{(1-r)^{q}}$ is monotone increasing on $\left[s^{-}, 0\right]$ and monotone decreasing on $\left[0, s^{+}\right]$. Hence, 
$\mathbf{B}_{\min }$ takes its minimum value at $r^{-}=s^{-}$and $\mathbf{B}_{\max }$ takes its maximum value at $r^{+}=0$, i.e.,

$$
x_{1}^{q} \leq \mathbf{B}_{\min }(x ; q, \delta) \leq \mathbf{B}_{\max }(x ; q, \delta) \leq \frac{\left(1-s^{+}\right)^{q}}{1-q s^{+}} x_{1}^{q} .
$$

If $0<q<1$, then the function $r \mapsto \frac{1-r q}{(1-r)^{q}}$ is monotone decreasing on $\left[s^{-}, 0\right]$ and monotone increasing on $\left[0, s^{+}\right]$. Hence, $\mathbf{B}_{\min }$ takes its minimum value at $r^{+}=0$ and $\mathbf{B}_{\max }$ takes its maximum value at $r^{-}=s^{-}$, i.e.,

$$
\frac{\left(1-s^{+}\right)^{q}}{1-q s^{+}} x_{1}^{q} \leq \mathbf{B}_{\min }(x ; q, \delta) \leq \mathbf{B}_{\max }(x ; q, \delta) \leq x_{1}^{q} .
$$

If $q<0$, then the function $r \mapsto \frac{1-r q}{(1-r)^{q}}$ is monotone increasing on $\left[s^{-}, 0\right]$ and monotone decreasing on $\left[0, s^{+}\right]$. Hence, $\mathbf{B}_{\min }$ takes its minimum value at $r^{+}=s^{+}$and $\mathbf{B}_{\max }$ takes its maximum value at $r^{-}=0$, i.e.,

$$
x_{1}^{q} \leq \mathbf{B}_{\min }(x ; q, \delta) \leq \mathbf{B}_{\max }(x ; q, \delta) \leq \frac{\left(1-s^{-}\right)^{q}}{1-q s^{-}} x_{1}^{q} .
$$

Corollary to Theorem $\mathbf{1}_{\infty}$. For the weights $w \in A_{\infty}^{\delta}(J)$, the Muckenhoupt condition $\left(A_{\mathbf{p}}\right)$ is fulfilled for all $\mathbf{p}>1-s^{-}$with the sharp constant

$$
\sup _{I \subset J}\langle w\rangle_{I}\left\langle w^{-\frac{1}{\mathbf{p}-1}}\right\rangle_{I}^{\mathbf{p}-1}=\frac{1}{\left(1-s^{-}\right)\left(1+\frac{s^{-}}{\mathbf{p}-1}\right)^{\mathbf{p}-1}} .
$$

To obtain this estimate, it suffices to apply Theorem $1_{\infty}$ with $q=-\frac{1}{\mathbf{p}-1}$. Comparison with the corollary to Theorem 1 shows that this result is the limit case of that statement as $p \rightarrow \infty$.

Outline of the proof of Theorem $\mathbf{2}_{\infty}$. We modify the arguments used in the proof of Theorem 2. As before, we denote by $B_{\max }(x ; q, \delta)$ and $B_{\min }(x ; q, \delta)$ the functions on the right-hand side of (30) and (31), respectively. The four lemmas for $p=\infty$ are the same as for $p \in(1, \infty)$. The only formal change occurs in Lemma $4_{\infty}$, where instead of the points $x^{ \pm}=\left(\langle w\rangle_{I^{ \pm}},\left\langle w^{1-p^{\prime}}\right\rangle_{I^{ \pm}}\right)$we must use $x^{ \pm}=\left(\langle w\rangle_{I^{ \pm}},\langle\log w\rangle_{I^{ \pm}}\right)$.

We shall not justify the limit passage as $p \rightarrow \infty$; it seems easier to prove Lemmas 1-4 for $p=\infty$ independently by the same method as for $p \in(1, \infty)$.

Proof of Lemma $1_{\infty}$. Since $x_{2}=\log x_{1}$ if and only if $w=x_{1}=$ const, it is clear that $\mathbf{B}\left(x_{1}, \log x_{1} ; q, \delta\right)=B_{ \pm}\left(x_{1}, \log x_{1} ; q, \delta\right)=x_{1}^{q}$ for all $x_{1}>0$ and all $q \in \mathbb{R}$. So, we need to consider $\delta>1$ and the points $x$ with $x_{2}<\log x_{1}$.

Fix an arbitrary point $x \in \Omega_{\delta}$ with $x_{2}<\log x_{1}$. Taking $a \in(0,1]$ and $s \in(-\infty, 1)$, we choose $J=[0,1]$ and put

$$
w_{a, s}(t)= \begin{cases}e^{x_{2}-s a} a^{s} t^{-s} & \text { if } 0 \leq t \leq a, \\ e^{x_{2}-s a} & \text { if } a \leq t \leq 1 .\end{cases}
$$

In Appendix 3 it will be shown that $w_{a, s} \in A_{\infty}^{\delta}(J)$ with $\delta=\frac{1}{1-s} e^{-s}$. Direct calculation yields $\left\langle\log w_{a, s}\right\rangle_{J}=x_{2}$. To get an extremal function for $\mathbf{B}$, we take

$$
\begin{aligned}
& s=s^{+}, a=1-\frac{r^{+}}{s^{+}} \quad \text { for } \mathbf{B}_{\max } \text { if } q>1 \text { and for } \mathbf{B}_{\min } \text { if } q<1, \\
& s=s^{-}, a=1-\frac{r^{-}}{s^{-}} \quad \text { for } \mathbf{B}_{\max } \text { if } q<1 \text { and for } \mathbf{B}_{\min } \text { if } q>1 .
\end{aligned}
$$


Recall that $s^{ \pm}=u^{ \pm}(1 / \delta)$, i.e., $\delta(1-s) e^{s}=1$, and $r^{ \pm}=u^{ \pm}\left(\frac{1}{\delta} x_{1} e^{-x_{2}}\right)$, i.e., $\delta(1-r) e^{r}=$ $x_{1} e^{-x_{2}}$. Since $\delta$ is assumed to be strictly greater than 1 , we have $s^{ \pm} \neq 0$, and $a$ is well defined. We check that $\left\langle w_{a, s}\right\rangle=x_{1}$ in all cases. Indeed,

$$
\begin{aligned}
\left\langle w_{a, s}\right\rangle_{J} & =\frac{1-(1-a) s}{1-s} e^{x_{2}-a s}=\delta(1-(1-a) s) e^{x_{2}+(1-a) s} \\
& =\delta e^{x_{2}}(1-r) e^{r}=\delta e^{x_{2}} \frac{1}{\delta} x_{1} e^{-x_{2}}=x_{1} .
\end{aligned}
$$

By (20), we have

$$
\left\langle w_{a, s}^{q}\right\rangle_{J}= \begin{cases}\frac{1-(1-a) q s}{1-q s} e^{q\left(x_{2}-a s\right)} & \text { if } q s<1 \\ \infty & \text { if } q s \geq 1 .\end{cases}
$$

Hence, for $q \geq 1 / s^{+}$and for $q \leq 1 / s^{-}$we get $\mathbf{B}_{\max }(x ; q, \delta)=B_{\max }(x ; q, \delta)=\infty$. And for $q s<1$ we have the identity

$$
\begin{aligned}
\left\langle w_{a, s}^{q}\right\rangle_{I} & =\frac{1-(1-a) q s}{1-q s}\left(\frac{1-s}{1-(1-a) s}\right)^{q}\left\langle w_{a, s}\right\rangle_{J}^{q} \\
& =\frac{1-q r}{1-q s}\left(\frac{1-s}{1-r}\right)^{q} x_{1}^{q}=B(x ; q, \delta) .
\end{aligned}
$$

As has already been mentioned, here we must take $s=s^{+}$and $r=r^{+}$for $B_{\max }(x ; q, \delta)$ if $q>1$ and for $B_{\min }(x ; q, \delta)$ if $q<1$, and $s=s^{-}, r=r^{-}$for $B_{\max }(x ; q, \delta)$ if $q<1$ and for $B_{\min }(x ; q, \delta)$ if $q>1$. Now, the required inequalities follow from the definition of $\mathbf{B}$.

Proof of Lemma $3_{\infty}$. To prove the lemma, we need to show that the matrix

$$
\left\{\frac{\partial^{2} B}{\partial x_{i} \partial x_{j}}\right\}_{i, j=1}^{2}
$$

is nonpositive for $B_{\max }$ and nonnegative for $B_{\min }$. Direct calculation yields

$$
\begin{aligned}
\frac{\partial r}{\partial x_{1}} & =-\frac{1-r}{r x_{1}}, \quad \frac{\partial r}{\partial x_{2}}=\frac{1-r}{r}, \\
\frac{\partial B}{\partial x_{1}} & =q^{2} x_{1}^{q-1} \frac{(1-s)^{q}}{(1-q s)(1-r)^{q-1}}, \\
\frac{\partial B}{\partial x_{2}} & =-q(q-1) x_{1}^{q} \frac{(1-s)^{q}}{(1-q s)(1-r)^{q}}, \\
\frac{\partial^{2} B}{\partial x_{1}^{2}} & =-q^{2}(q-1) x_{1}^{q-2} \frac{(1-s)^{q}}{(1-q s) r(1-r)^{q-2}}, \\
\frac{\partial^{2} B}{\partial x_{1} \partial x_{2}} & =q^{2}(q-1) x_{1}^{q-1} \frac{(1-s)^{q}}{(1-q s) r(1-r)^{q-1}}, \\
\frac{\partial^{2} B}{\partial x_{2}^{2}} & =-q^{2}(q-1) x_{1}^{q} \frac{(1-s)^{q}}{(1-q s) r(1-r)^{q}} .
\end{aligned}
$$

Therefore, the quadratic form of the matrix (32) is

$$
\sum_{i, j=1}^{2} \frac{\partial^{2} B}{\partial x_{i} \partial x_{j}} \Delta_{i} \Delta_{j}=-\frac{q^{2}(q-1) x_{1}^{q-2}}{r(1-q s)}\left(\frac{1-s}{1-r}\right)^{q}\left((1-r) \Delta_{1}-x_{1} \Delta_{2}\right)^{2} .
$$

This expression has the required sign, because the quantity $1-q s$ is always positive and the sign of $r$ coincides with the sign of $q-1$ for $B_{\max }$ and is opposite to the latter for $B_{\min }$. 
Proof of Lemma $4_{\infty}$. This is a word-for-word repetition of the proof of Lemma 4 . We must only replace the expression $x_{1} x_{2}^{p-1}$ by $x_{1} e^{-x_{2}}$. The equation of the line tangent to the graph $x_{1} e^{-x_{2}}=\varepsilon$ at a point $\tau$ is $x_{1}-\tau_{1} x_{2}=\tau_{1}\left(1-\tau_{2}\right)$. This line intersects the graph $x_{1} e^{-x_{2}}=\delta$ at the points

$$
x(\delta)^{ \pm}=\left(\tau_{1}\left[1-u_{\infty}^{ \pm}\left(\frac{\delta}{\varepsilon}\right)\right], \tau_{2}-u_{\infty}^{ \pm}\left(\frac{\delta}{\varepsilon}\right)\right) .
$$

Finally, we get the same estimate

$$
\min \alpha^{ \pm} \geq \frac{\left|u_{\infty}^{+}(\delta / \varepsilon)-u_{\infty}^{-}(\delta / \varepsilon)\right|}{\left|u_{\infty}^{+}(1 / \varepsilon)-u_{\infty}^{-}(1 / \varepsilon)\right|}
$$

Proof of Lemma $2_{\infty}$. It also follows the lines of the proof of Lemma 2. Here the changes are even slighter; namely, $x_{2}=\left\langle w^{1-p^{\prime}}\right\rangle_{I}$ must be replaced by $x_{2}=\langle\log w\rangle_{I}$.

We pass to the other limit case: $p=1$. Now the changes to be made are far more extensive. To any weight $w$ in $A_{1}^{\delta}(I)$, we assign the point $x=\left(x_{1}, x_{2}\right)=\left(\langle w\rangle_{I}, \sup _{I} 1 / w\right)$; these points fill the domain

$$
\Omega_{\delta}(1) \stackrel{\text { def }}{=}\left\{\left(x_{1}, x_{2}\right): x_{2} \in \mathbb{R}, 1 \leq x_{1} x_{2} \leq \delta\right\}
$$

as the weight $w$ runs over $A_{1}^{\delta}(I)$. On $\Omega_{\delta}(1)$ we define the following functions:

$$
\begin{aligned}
& \mathbf{B}_{\max }(x ; q, \delta) \\
& \quad=\mathbf{B}_{\max }(x ; 1, q, \delta) \stackrel{\text { def }}{=} \sup _{w \in A_{\infty}^{\delta}(I)}\left\{\left\langle w^{q}\right\rangle_{I}:\langle w\rangle_{I}=x_{1}, \sup _{I} \frac{1}{w}=x_{2}\right\}, \\
& \mathbf{B}_{\min }(x ; q, \delta) \\
& \quad=\mathbf{B}_{\min }(x ; 1, q, \delta) \stackrel{\text { def }}{=} \inf _{w \in A_{\infty}^{\delta}(I)}\left\{\left\langle w^{q}\right\rangle_{I}:\langle w\rangle_{I}=x_{1}, \sup _{I} \frac{1}{w}=x_{2}\right\} .
\end{aligned}
$$

To compare the expressions for $\mathbf{B}(x ; p, q, \delta)$ with $p \in(1, \infty)$ and $\mathbf{B}(x ; 1, q, \delta)$, we now replace $x_{2}^{p-1}$ with $x_{2}$ and then pass to the limit as $p \rightarrow 1$. It should be noted that if $p=1+\varepsilon, \varepsilon \rightarrow 0$, then asymptotically we have

$$
\begin{aligned}
s^{+} & \approx 1-\frac{\varepsilon}{\delta-1}, & s^{-} & \approx-\delta^{\frac{1}{\varepsilon}}, \\
r^{+} & \approx 1-\frac{\varepsilon x_{1} x_{2}}{\delta-x_{1} x_{2}}, & r^{-} & \approx-\left(\frac{\delta}{x_{1} x_{2}}\right)^{\frac{1}{\varepsilon}} .
\end{aligned}
$$

Limit passage as $p \rightarrow 1$ in (13)-(14) provides the expression for $\mathbf{B}(x ; 1, q, \delta)$. In Appendix 1 it will be explained how to find these expressions from the Bellman equation. Now we state the result.

Theorem 2 $2_{1}$ For $x_{1} x_{2}=1$ we have

$$
\mathbf{B}_{\max }(x ; p, q, \delta)=\mathbf{B}_{\min }(x ; p, q, \delta)=x_{1}^{q} .
$$

Otherwise

$$
\mathbf{B}_{\max }(x ; q, \delta)= \begin{cases}+\infty & \text { if } q \in\left[\frac{\delta}{\delta-1},+\infty\right), \\ x_{2}^{-q} \frac{q x_{1} x_{2}-(q-1) \delta}{q-(q-1) \delta} & \text { if } q \in(-\infty, 0] \cup\left[1, \frac{\delta}{\delta-1}\right), \\ x_{1}^{q} & \text { if } q \in[0,1],\end{cases}
$$


and

$$
\mathbf{B}_{\min }(x ; q, \delta)= \begin{cases}x_{1}^{q} & \text { if } q \in(-\infty, 0) \cup[1,+\infty), \\ x_{2}^{-q} \frac{q x_{1} x_{2}-(q-1) \delta}{q-(q-1) \delta} & \text { if } q \in[0,1] .\end{cases}
$$

Before proving this theorem, we deduce Theorem $1_{1}$ from it. If $\mathbf{B}=x_{1}^{q}$, there is nothing to prove; we consider the case where

$$
\mathbf{B}=x_{2}^{-q} \frac{q x_{1} x_{2}-(q-1) \delta}{q-(q-1) \delta} .
$$

Then

$$
\mathbf{B}(x) x_{1}^{-q}=y^{-q} \frac{q y-(q-1) \delta}{q-(q-1) \delta}, \quad y=x_{1} x_{2} \in[1, \delta] .
$$

We calculate the derivative of the function $y \mapsto y^{-q}(q y-(q-1) \delta)$ :

$$
\frac{\partial}{\partial y}\left(q y^{-q+1}-(q-1) \delta y^{-q}\right)=q(q-1) y^{-q-1}(\delta-y) \text {. }
$$

Therefore, this function is monotone increasing if $q \notin[0,1]$ and monotone decreasing otherwise. Since at the point $y=\delta$ this function is equal to $\delta^{1-q}$, we obtain the constant $C_{\max }=\delta^{1-q}(q-(q-1) \delta)^{-1}$ if $q \notin[0,1]$ and the same expression for $C_{\min }$ for $q \in(0,1)$.

Corollary to Theorem $\mathbf{1}_{\mathbf{1}}$. For the weights $w, w \in A_{1}^{\delta}(J)$, the Muckenhoupt condition $\left(A_{\mathbf{p}}\right)$ is fulfilled for all $\mathbf{p}>1$ with the sharp constant

$$
\sup _{I \subset J}\langle w\rangle_{I}\left\langle w^{-\frac{1}{\mathbf{p}-1}}\right\rangle_{I}^{\mathbf{p}-1}=\frac{\delta^{\mathbf{p}}}{\left(1+\mathbf{p}^{\prime}(\delta-1)\right)^{\mathbf{p}-1}} .
$$

To obtain this formula, it suffices to apply Theorem $1_{1}$ with $q=1-\mathbf{p}^{\prime}$. The result is easily seen to be the limit case of the corollary to Theorem 1 as $p \rightarrow 1$. To verify this, we use the asymptotic behavior of $s^{+}$and $r^{+}$mentioned above for obtaining the following two limit relations:

$$
\begin{gathered}
\frac{1-\frac{s^{+}}{p}}{1-s^{+}} \rightarrow \delta, \\
\frac{1-\frac{\mathbf{p}-p}{p(\mathbf{p}-1)} s^{+}}{1-s^{+}}=1+\mathbf{p}^{\prime} \frac{p-1}{p\left(1-s^{+}\right)} \rightarrow 1+\mathbf{p}^{\prime}(\delta-1) .
\end{gathered}
$$

Therefore,

$$
\frac{\left(1-\frac{s}{p}\right)^{\mathbf{p}}}{(1-s)\left(1-\frac{\mathbf{p}-p}{p(\mathbf{p}-1)} s\right)^{\mathbf{p}-1}} \rightarrow \frac{\delta^{\mathbf{p}}}{\left(1+\mathbf{p}^{\prime}(\delta-1)\right)^{\mathbf{p}-1}} .
$$

Outline of the proof of Theorem 21. Again we shall use the method of the proof of Theorem 2. As in that theorem, we denote by $B_{\max }(x ; q, \delta)$ and $B_{\min }(x ; q, \delta)$ the functions on the right-hand sides of (36) and (37), respectively. Now only the statements of the first two lemmas for $p=1$ remain the same as for $p \in(1, \infty)$.

Two other lemmas must be modified for the following reasons. Before, when splitting an interval $I$ into two parts $I=I^{-} \cup I^{+}$, we had a proportional splitting of the interval $\left[x^{-}, x^{+}\right]\left(x^{ \pm}=\left(\langle w\rangle_{I^{ \pm}},\left\langle w^{1-p^{\prime}}\right\rangle_{I^{ \pm}}\right)\right)$by the point $x^{0}=\left(\langle w\rangle_{I},\left\langle w^{1-p^{\prime}}\right\rangle_{I}\right)$. But now this is not the case, and the point $x^{0}$ may even fail to be on the interval with the endpoints $x^{-}$ and $x^{+}$. In the situation under study, a proportional splitting arises only for the first coordinate $x_{1}^{0}=\alpha^{-} x_{1}^{-}+\alpha^{+} x_{1}^{+}$, whereas for the second coordinate we have

$$
x_{2}^{0}=\sup _{I} \frac{1}{w}=\max \left\{\sup _{I^{-}} \frac{1}{w}, \sup _{I^{+}} \frac{1}{w}\right\}=\max \left\{x_{2}^{-}, x_{2}^{+}\right\} .
$$


For this reason, for $p=1$ we restate Lemma 3 as follows.

Lemma $3_{1}$. Let $x^{ \pm}$be two arbitrary points in $\Omega_{\delta}$, and let $\alpha^{ \pm}$be a pair of nonnegative numbers such that $\alpha^{-}+\alpha^{+}=1$. Put $x^{0}=\left(x_{1}^{0}, x_{2}^{0}\right) \stackrel{\text { def }}{=}\left(\alpha^{-} x_{1}^{-}+\alpha^{+} x_{1}^{+}, \max \left\{x_{2}^{-}, x_{2}^{+}\right\}\right)$. If both points $\left(x_{1}^{ \pm}, x_{2}^{0}\right)$ are in $\Omega_{\delta}$, then

$$
\begin{aligned}
B_{\max }\left(x^{0}\right) & \geq \alpha^{-} B_{\max }\left(x^{-}\right)+\alpha^{+} B_{\max }\left(x^{+}\right), \\
B_{\min }\left(x^{0}\right) & \leq \alpha^{-} B_{\min }\left(x^{-}\right)+\alpha^{+} B_{\min }\left(x^{+}\right) .
\end{aligned}
$$

Lemma 4 must be changed as follows.

Lemma $4_{1}$. If a parameter $\delta>1$ is given, then for an arbitrary $\varepsilon>\delta$ and an arbitrary weight $w \in A_{1}^{\delta}(I)$ there exists a splitting $I=I^{-} \cup I^{+},\left|I^{ \pm}\right|=\alpha^{ \pm}|I|$, such that $x_{1}^{ \pm} x_{2}^{0} \leq$ $\varepsilon$, where $x^{ \pm}=\left(\langle w\rangle_{I^{ \pm}},\left\langle w^{1-p^{\prime}}\right\rangle_{I^{ \pm}}\right)$and $x^{0}=\left(\langle w\rangle_{I},\left\langle w^{1-p^{\prime}}\right\rangle_{I}\right)$. Moreover, the splitting parameters $\alpha^{ \pm}$can be chosen bounded away from 0 to 1 , uniformly with respect to $w$ and, therefore, with respect to $I$.

Proof of Lemma $1_{1}$. Now the cases of $\mathbf{B}=x_{2}^{-q} \frac{q x_{1} x_{2}-(q-1) \delta}{q-(q-1) \delta}$ and of $\mathbf{B}=x_{1}^{q}$ are entirely different. In the first case, we can take the limit (as $p \rightarrow 1$ ) of the expression for the extremal function we already know:

$$
w_{c, a, \nu}(t)= \begin{cases}c a^{\nu} t^{-\nu} & \text { if } 0 \leq t \leq a, \\ c & \text { if } a \leq t \leq 1,\end{cases}
$$

with the limit values of the parameters in (21) for $s=s^{+}$and $r=r^{+}$:

$$
\nu=\frac{\delta-1}{\delta}, \quad a=\frac{x_{1} x_{2}-1}{\delta-1}, \quad c=\frac{1}{x_{2}} .
$$

We check that this weight $w$ is in $A_{1}^{\delta}$. For any monotone decreasing weight $w$, we have

$$
\begin{aligned}
& \sup _{0 \leq \alpha<\beta \leq 1}\left\{\langle w\rangle_{(\alpha, \beta)} \cdot \sup _{(\alpha, \beta)} \frac{1}{w}\right\}=\sup _{0 \leq \alpha<\beta \leq 1}\left\{\langle w\rangle_{(\alpha, \beta)} \cdot \frac{1}{w(\beta)}\right\} \\
& =\sup _{0 \leq \beta \leq 1}\left\{\langle w\rangle_{(0, \beta)} \cdot \frac{1}{w(\beta)}\right\} .
\end{aligned}
$$

Since our weight satisfies $w(\beta)=$ const for $\beta>a$, we have

$$
\sup _{I \subset[0,1]}\left\{\langle w\rangle_{I} \cdot \sup _{I} \frac{1}{w}\right\}=\sup _{0 \leq \beta \leq a}\left\{\langle w\rangle_{(0, \beta)} \cdot \frac{1}{w(\beta)}\right\} .
$$

Furthermore, $\langle w\rangle_{(0, \beta)}=\frac{c}{1-\nu}(a / \beta)^{\nu}$ if $\beta \leq a$, whence

$$
\sup _{I \subset[0,1]}\left\{\langle w\rangle_{I} \cdot \sup _{I} \frac{1}{w}\right\}=\sup _{0 \leq \beta \leq a}\left\{\frac{c}{1-\nu}\left(\frac{a}{\beta}\right)^{\nu} \cdot \frac{1}{c}\left(\frac{\beta}{a}\right)^{\nu}\right\}=\frac{1}{1-\nu}=\delta .
$$

Now, we check that the weight $w$ represents the point $x=\left(x_{1}, x_{2}\right)$ in $\Omega_{\delta}(1)$ :

$$
\begin{aligned}
\langle w\rangle_{[0,1]} & =\frac{c a}{1-\nu}+(1-a) c=x_{1}, \\
\sup _{[0,1]} \frac{1}{w} & =x_{2} .
\end{aligned}
$$


Thus, for $q \geq \frac{\delta}{\delta-1}$ we get $\mathbf{B}_{\max }(x ; q, \delta)=B_{\max }(x ; q, \delta)=\infty$. For $q<\frac{\delta}{\delta-1}$, substituting $\theta=q$ and the chosen values of $\nu, a$, and $c$ in (20), we obtain

$$
\begin{aligned}
\left\langle w^{q}\right\rangle_{[0,1]} & =c^{q} \frac{1-(1-a) q \nu}{1-q \nu}=x_{2}^{-q} \frac{1-\frac{\delta-x_{1} x_{2}}{\delta-1} q \frac{\delta-1}{\delta}}{1-q \frac{\delta-1}{\delta}} \\
& =x_{2}^{-q} \frac{q x_{1} x_{2}-\delta(q-1)}{q-\delta(q-1)}=B(x ; q, \delta),
\end{aligned}
$$

which implies the inequalities of Lemma $1_{1}$ for $\mathbf{B}_{\max }, q \notin[0,1]$ and for $\mathbf{B}_{\min }, q \in(0,1)$.

The case of $\mathbf{B}=x_{1}^{q}$ looks more difficult, because the relation $\left\langle w^{q}\right\rangle=\langle w\rangle^{q}=x_{1}^{q}$ occurs if and only if $w$ is a constant function, and this means that there is no extremal function for an arbitrary point of $\Omega_{\delta}(1)$ except the boundary $x_{1} x_{2}=1$. Nevertheless, we can construct a family of weights in $A_{1}^{\delta}$ representing a given point $\left(x_{1}, x_{2}\right)$ and approximating the maximum or minimum value of $\left\langle w^{q}\right\rangle$. Using the weight $w$ constructed above, consider the following family of weights $\left\{w_{\lambda}\right\}_{0<\lambda<1}$ :

$$
w_{\lambda}(t)= \begin{cases}\langle w\rangle_{(0, \lambda)} & \text { if } 0 \leq t \leq \lambda \\ w(t) & \text { if } \lambda<t \leq 1\end{cases}
$$

For every monotone decreasing weight $w$ on $[0,1]$, we introduce the following function on the same interval:

$$
\varphi_{w}(\beta)=\langle w\rangle_{(0, \beta)} \cdot \frac{1}{w(\beta)} .
$$

Clearly,

$$
\varphi_{w_{\lambda}}(\beta)= \begin{cases}1 & \text { if } 0 \leq \beta \leq \lambda \\ \varphi_{w}(\beta) & \text { if } \lambda<\beta \leq 1\end{cases}
$$

By (40), the $A_{1^{-}}$"norm" of $w$ is equal to the supremum of the function $\varphi_{w}$; therefore, the $A_{1^{-}}$"norm" of all $w_{\lambda}$ never exceeds the $A_{1^{-}}$"norm" of $w$, i.e., $w_{\lambda} \in A_{1}^{\delta}$. It is clear that all $w_{\lambda}$ represent the same point $\left(x_{1}, x_{2}\right)$ because neither the average of the weight nor its minimum value changes under the construction of $w_{\lambda}$. But the functions $w_{\lambda}$ tend almost everywhere to the constant function $w_{1}(t)=x_{1}$ as $\lambda \rightarrow 1$. Therefore, $\left\langle w_{\lambda}^{q}\right\rangle_{[0,1]} \rightarrow x_{1}^{q}$, i.e., $\left\{w_{\lambda}\right\}$ is the required approximating family of weights.

Remark. Note that Lemma $1_{1}$ completes the proof of Theorem $2_{1}$ not only in the evident case where $\mathbf{B}_{\max }=\infty$, but also in the case where $\mathbf{B}=x_{1}^{q}$, because in this case the reverse inequalities are valid for an arbitrary weight $w$ :

$$
\begin{cases}\left\langle w^{q}\right\rangle \geq\langle w\rangle^{q} & \text { if } q \notin(0,1), \\ \left\langle w^{q}\right\rangle \leq\langle w\rangle^{q} & \text { if } q \in[0,1] .\end{cases}
$$

Indeed, for $q>1$ this is the Hölder inequality applied to the product $w \cdot 1$ with the exponent $q$, for $0<q<1$ this is the Hölder inequality applied to the product $w^{q} \cdot 1$ with the exponent $1 / q$, and for $q<0$ this is the Hölder inequality applied to the product $1=w^{\frac{q}{q-1}} \cdot w^{\frac{q}{1-q}}$ with the exponent $\frac{q-1}{q}$.

Proof of Lemma $3_{1}$. In spite of the fact that we do not need this lemma in the case where $B(x)=x_{1}^{q}$ (see the above remark), the lemma is obvious in this case because $B(x)$ depends only of $x_{1}$, and with respect to this variable the function is concave for $q \notin[0,1]$ and convex for $q \in(0,1)$. So, in the remaining part of the proof we shall consider the case where $B(x)=x_{2}^{-q} \frac{q x_{1} x_{2}-(q-1) \delta}{q-(q-1) \delta}$. 
Since the roles of the points $x^{ \pm}$are symmetric, there is no loss of generality in assuming that, say, $x_{2}^{+}=\max \left\{x_{2}^{ \pm}\right\}=x_{2}^{0}$. Then, since $B$ is linear in $x_{1}$, we have

$$
\begin{aligned}
B\left(x^{0}\right) & -\left(\alpha^{-} B\left(x^{-}\right)+\alpha^{+} B\left(x^{+}\right)\right) \\
= & \left(\alpha^{-} B\left(x_{1}^{-}, x_{2}^{+}\right)+\alpha^{+} B\left(x_{1}^{+}, x_{2}^{+}\right)\right)-\left(\alpha^{-} B\left(x_{1}^{-}, x_{2}^{-}\right)+\alpha^{+} B\left(x_{1}^{+}, x_{2}^{+}\right)\right) \\
= & \alpha^{-}\left(B\left(x_{1}^{-}, x_{2}^{+}\right)-B\left(x_{1}^{-}, x_{2}^{-}\right)\right) .
\end{aligned}
$$

The sign of this expression coincides with the sign of the derivative

$$
\frac{\partial B\left(x_{1}^{-}, x_{2}\right)}{\partial x_{2}}=q(q-1) x_{2}^{-q-1} \frac{\delta-x_{1}^{-} x_{2}}{q-(q-1) \delta}
$$

which coincides with the sign of $q(q-1)$ because by assumption the point $\left(x_{1}^{-}, x_{2}^{+}\right)$(and, therefore, the entire interval $\left.\left\{\left(x_{1}^{-}, x_{2}\right): x_{2}^{-} \leq x_{2} \leq x_{2}^{+}\right\}\right)$is in $\Omega_{\delta}$, i.e., $x_{1}^{-} x_{2} \leq \delta$. This implies inequality (38) for $q \notin[0,1]$ and inequality (39) for $q \in(0,1)$.

Proof of Lemma $4_{1}$. Without loss of generality we may assume that $\varepsilon<2 \delta$, because if the lemma is true for some $\varepsilon$, it is also true for all larger $\varepsilon$. Since $x_{2}^{0}=\max \left\{x_{2}^{ \pm}\right\}$and both points $x^{ \pm}$are in $\Omega_{\delta}$, at least one of the inequalities $x_{1}^{ \pm} x_{2}^{0} \leq \varepsilon$ is always true. First we take $\alpha^{-}=\alpha^{+}=1 / 2$; if the required inequalities are both true, we fix this splitting. Otherwise, we start to change $\alpha$, namely, we increase $\alpha^{+}$if the point $\left(x_{1}^{+}, x_{2}^{0}\right)$ is outside $\Omega_{\varepsilon}$ and reduce it (i.e., increase $\alpha^{-}$) in the opposite case. By symmetry, it suffices to examine one of the possible situations, say, the case where $x_{1}^{+} x_{2}^{0}>\varepsilon$ for $\alpha^{+}=1 / 2$. So, we begin to enlarge the interval $I^{+}$(i.e., to increase $\alpha^{+}$) and stop at the moment when $x_{1}^{+} x_{2}^{0}=\varepsilon$ for the first time. This will be our splitting procedure. Now we must check that such a moment does exist, and, moreover, the corresponding value of $\alpha^{+}$is bounded away from 0 and 1 uniformly with respect to $w$ and $I$.

For $p=1$ the points $x^{ \pm}$do not depend continuously on $\alpha^{+}$in general, but the first coordinates $x_{1}^{ \pm}$do. We check that $x_{1}^{+} x_{2}^{0}<\varepsilon$ for $\alpha^{+}=\delta / \varepsilon$, which implies that the required value of $\alpha^{+}$exists and is in $(1 / 2, \delta / \varepsilon)$. Since $x_{1}^{0}=\alpha^{-} x_{1}^{-}+\alpha^{+} x_{1}^{+}$, we have

$$
x_{1}^{+}<\frac{x_{1}^{0}}{\alpha^{+}}=\frac{x_{1}^{0} x_{2}^{0}}{\alpha^{+} x_{2}^{0}} \leq \frac{\delta}{\alpha^{+} x_{2}^{0}},
$$

whence for $\alpha^{+}=\delta / \varepsilon$ we get

So, we can conclude that

$$
x_{1}^{+} x_{2}^{0}<\frac{\delta}{\alpha^{+}}=\varepsilon
$$

$$
\max \left\{\alpha^{ \pm}\right\} \leq \frac{\delta}{\varepsilon}
$$

and this estimate only depends on $\delta$ and $\varepsilon$, but not on the choice of the interval $I$ or the weight $w$.

Proof of Lemma $22_{1}$. It follows the lines of the proof of Lemma 2. Here only $x_{2}=\left\langle w^{1-p^{\prime}}\right\rangle_{I}$ must be replaced by $x_{2}=\sup _{I} 1 / w$.

Appendix 1: how to find the Bellman functions (13)-(14), (30)-(31), and (36)-(37). First, consider the case where $1<p<\infty$. We begin with explaining why we look for a concave function $\mathbf{B}_{\max }$ and a convex function $\mathbf{B}_{\min }$. If an interval $I$ is split into two parts $I=I^{+} \cup I^{-},\left|I^{ \pm}\right|=\alpha^{ \pm}|I|$, we consider two extremal weights $w^{ \pm}$ (or "almost extremal" if there is no extremal function) defined on $I^{ \pm}$, respectively, and write the identity

$$
\left\langle w^{q}\right\rangle_{I}=\alpha^{-}\left\langle w^{q}\right\rangle_{I^{-}}+\alpha^{+}\left\langle w^{q}\right\rangle_{I^{+}}
$$

for the weight $w$ on $I$ coinciding with $w^{+}$on $I^{+}$and with $w^{-}$on $I^{-}$. We see that on the right-hand side we have a convex combination of the values of the Bellman function $\mathbf{B}$ at 
the points $x^{ \pm}$(or almost these values if there is no extremal function), but the average on the left-hand side is between $\mathbf{B}_{\min }\left(x^{0}\right)$ and $\mathbf{B}_{\max }\left(x^{0}\right), x^{0}=\alpha^{-} x^{-}+\alpha^{+} x^{+}$, by the definition of the Bellman function. This leads to the concavity condition for $\mathbf{B}_{\max }$ and to the convexity condition for $\mathbf{B}_{\min }$. Of course, it is not quite clear why the above weight $w$ is in $A_{p}^{\delta}$ on the entire interval $I$. However, the heuristic nature of the forthcoming arguments allows us to drop this question.

Next, we check that the functions $\mathbf{B}$ must be of the following form:

$$
\mathbf{B}\left(x_{1}, x_{2} ; p, q, \delta\right)=x_{1}^{q} g\left(x_{1} x_{2}^{p-1} ; p, q, \delta\right),
$$

where $g(\cdot ; p, q, \delta)$ is a function on $[1, \delta]$ such that $g \geq 1$ for $q \notin(0,1), 0 \leq g \leq 1$ for $q \in[0,1]$, and the boundary condition $g(1 ; p, q, \delta)=1$ is satisfied.

Indeed, if a weight $w$ runs over the entire class $A_{p}^{\delta}(J)$, then so does the weight $\tilde{w}=t w$, where $t$ is an arbitrary positive constant. For the averages of these weights we have $\langle\tilde{w}\rangle=t\langle w\rangle,\left\langle\tilde{w}^{1-p^{\prime}}\right\rangle=t^{1-p^{\prime}}\left\langle w^{1-p^{\prime}}\right\rangle$, and $\left\langle\tilde{w}^{q}\right\rangle=t^{q}\left\langle w^{q}\right\rangle$. Taking the supremum (or infimum) in the latter identity, we get

$$
\mathbf{B}\left(t x_{1}, t^{1-p^{\prime}} x_{2} ; p, q, \delta\right)=t^{q} \mathbf{B}\left(x_{1}, x_{2} ; p, q, \delta\right) .
$$

Putting $t=x_{2}^{p-1}$, we obtain the relation

$$
\mathbf{B}\left(x_{1} x_{2}^{p-1}, 1 ; p, q, \delta\right)=x_{2}^{q(p-1)} \mathbf{B}\left(x_{1}, x_{2} ; p, q, \delta\right) .
$$

Therefore, $\mathbf{B}(x)=x_{1}^{q} g\left(x_{1} x_{2}^{p-1}\right)$, where $g$ is a function on $[1, \delta]$ defined by the identity $g(y)=y^{-q} \mathbf{B}(y, 1)$. By the Hölder inequality we have $\langle w\rangle^{q} \leq\left\langle w^{q}\right\rangle$ for $q \notin(0,1)$ and $\langle w\rangle^{q} \geq\left\langle w^{q}\right\rangle$ for $q \in[0,1]$ (see (41)). Therefore, $\mathbf{B}(x) \geq x_{1}^{q}$ for $q \notin(0,1)$ and $\mathbf{B}(x) \leq x_{1}^{q}$ for $q \in[0,1]$, which implies that $g \geq 1$ for $q \notin(0,1)$ and $0 \leq g \leq 1$ for $q \in[0,1]$. Moreover, since the relation $x_{2}=x_{1}^{1-p^{\prime}}$ occurs if and only if $w=x_{1}=$ const, we have $\mathbf{B}\left(x_{1}, x_{1}^{1-p^{\prime}} ; p, q, \delta\right)=x_{1}^{q}$, i.e., $g(1 ; p, q, \delta)=1$.

Now, we introduce the variable $y=x_{1} x_{2}^{p-1}$ and calculate the matrix of the second derivatives (23) for the "true" Bellman function B in terms of $g$. Using the representation (42), we obtain

$$
\begin{aligned}
& \frac{\partial y}{\partial x_{1}}=\frac{y}{x_{1}}, \quad \frac{\partial y}{\partial x_{2}}=(p-1) \frac{y}{x_{2}} \\
& \frac{\partial \mathbf{B}}{\partial x_{1}}=q x_{1}^{q-1} g+x_{1}^{q} g^{\prime} \frac{\partial y}{\partial x_{1}}=x_{1}^{q-1}\left(q g+y g^{\prime}\right), \\
& \frac{\partial \mathbf{B}}{\partial x_{2}}=x_{1}^{q} g^{\prime} \frac{\partial y}{\partial x_{2}}=(p-1) x_{1}^{q} x_{2}^{-1} y g^{\prime}, \\
& \frac{\partial^{2} \mathbf{B}}{\partial x_{1}^{2}}=(q-1) x_{1}^{q-2}\left(q g+y g^{\prime}\right)+x_{1}^{q-1}\left(q g^{\prime}+g^{\prime}+y g^{\prime \prime}\right) \frac{\partial y}{\partial x_{1}} \\
&=x_{1}^{q-2}\left(q(q-1) g+2 q y g^{\prime}+y^{2} g^{\prime \prime}\right), \\
& \frac{\partial^{2} \mathbf{B}}{\partial x_{1} \partial x_{2}}=x_{1}^{q-1}\left(q g^{\prime}+g^{\prime}+y g^{\prime \prime}\right) \frac{\partial y}{\partial x_{2}}=(p-1) x_{1}^{q-1} x_{2}^{-1}\left((q+1) y g^{\prime}+y^{2} g^{\prime \prime}\right), \\
& \frac{\partial^{2} \mathbf{B}}{\partial x_{2}^{2}}=-(p-1) x_{1}^{q} x_{2}^{-2} y g^{\prime}+(p-1) x_{1}^{q} x_{2}^{-1}\left(g^{\prime}+y g^{\prime \prime}\right) \frac{\partial y}{\partial x_{2}} \\
&=(p-1) x_{1}^{q} x_{2}^{-2}\left((p-2) y g^{\prime}+(p-1) y^{2} g^{\prime \prime}\right),
\end{aligned}
$$

and the matrix (23) turns into

$$
\left(\begin{array}{cc}
\frac{\partial^{2} \mathbf{B}}{\partial x_{1}^{2}} & \frac{\partial^{2} \mathbf{B}}{\partial x_{1} \partial x_{2}} \\
\frac{\partial^{2} \mathbf{B}}{\partial x_{1} \partial x_{2}} & \frac{\partial^{2} \mathbf{B}}{\partial x_{2}^{2}}
\end{array}\right)=\frac{x_{1}^{q-2}}{p-1}\left(\begin{array}{cc}
1 & 1 \\
0 & (p-1) x_{1} x_{2}^{-1}
\end{array}\right) R\left(\begin{array}{cc}
1 & 0 \\
1 & (p-1) x_{1} x_{2}^{-1}
\end{array}\right),
$$


where

$$
R=\left(\begin{array}{cc}
p q(\gamma-1) g-p y g^{\prime} & p \gamma y g^{\prime} \\
p \gamma y g^{\prime} & (p-2) y g^{\prime}+(p-1) y^{2} g^{\prime \prime}
\end{array}\right) .
$$

If this matrix were nonsingular, equality in (18)-(19) could not occur except for the case where $x^{-}=x^{+}$. However, for an extremal function $w$ for $\mathbf{B}_{\max }$ (if such a function exists) we have

$\mathbf{B}_{\max }\left(x^{0}\right)=\left\langle w^{q}\right\rangle_{I}=\alpha^{-}\left\langle w^{q}\right\rangle_{I^{-}}+\alpha^{+}\left\langle w^{q}\right\rangle_{I^{+}} \leq \alpha^{-} \mathbf{B}_{\max }\left(x^{-}\right)+\alpha^{+} \mathbf{B}_{\max }\left(x^{+}\right) \leq \mathbf{B}_{\max }\left(x^{0}\right)$.

So, for a nonsingular matrix (43) this leads to the identity $x^{+}=x^{-}$for every splitting of the initial interval $I$, which means that the weight $w$ is constant. Since for any point of $\Omega_{\delta}$ except for the boundary $x_{1} x_{2}^{p-1}=1$ this is not the case, the matrix (43) must be singular.

It is possible to analyze the situation more carefully in the case where there is no extremal weight and we have only a sequence of weights whose $q$ th powers tend to the value of $\mathbf{B}$, but we omit this, again by the heuristic nature of the arguments in this appendix: the said above already justifies looking for a singular matrix (43). So, we equate the determinant of the matrix (44) to zero:

$$
\left(q(\gamma-1) g-y g^{\prime}\right)\left((p-2) y g^{\prime}+(p-1) y^{2} g^{\prime \prime}\right)=p\left(\gamma y g^{\prime}\right)^{2} .
$$

The concavity condition for $\mathbf{B}_{\max }$ and the convexity condition for $\mathbf{B}_{\min }$ take the form

$$
y g_{\min }^{\prime}-q(\gamma-1) g_{\min } \leq 0 \leq y g_{\max }^{\prime}-q(\gamma-1) g_{\max } .
$$

Let us solve equation (45). We introduce a new variable $h=y g^{\prime} / g$. Then $y^{2} g^{\prime \prime} / g=$ $y h^{\prime}+h^{2}-h$, and (45) takes the form

$$
(p q(\gamma-1)-p h)\left((p-1)\left(h^{2}+y h^{\prime}\right)-h\right)=(p \gamma h)^{2} ;
$$

therefore,

$$
\begin{gathered}
(p-1) y h^{\prime}(q(q-1)(p-1)-p h) \\
=(p q-q+1)^{2} h^{2}-(q(q-1)(p-1)-p h)\left((p-1) h^{2}-h\right) \\
=p(p-1) h^{3}+\left(p^{2} q-p-q+1\right) h^{2}+q(q-1)(p-1) h \\
=(p-1) h(h+q)(p h+q-1), \\
\frac{q(q-1)(p-1)-p h}{h(h+q)(p h+q-1)} d h=\frac{d y}{y}, \\
\left(\frac{p-1}{h}+\frac{1}{h+q}-\frac{p^{2}}{p h+q-1}\right) d h=\frac{d y}{y}, \\
(p-1) \log |h|+\log |h+q|-p \log |p h+q-1|=\log |y|+\text { const, } \\
y=C \frac{|h+q||h|^{p-1}}{|p h+q-1|^{p}} .
\end{gathered}
$$

Since $g>0$, (46) can be rewritten as follows:

$$
h_{\min } \leq q(\gamma-1) \leq h_{\max } .
$$

Now we introduce a more convenient variable $r$ by the formula

$$
r=\frac{h-q(\gamma-1)}{\gamma h} \quad \text { or } \quad h=\frac{q(\gamma-1)}{1-\gamma r} .
$$

Rewriting (47) in terms of $r$, we get

$$
y=\delta \frac{1-r}{(1-r / p)^{p}},
$$


where $\delta$ is a new constant parameter, so that $r=r_{p}^{ \pm}=u_{p}^{ \pm}(y / \delta)$. The new parameter is denoted by $\delta$ because then the natural domain where $g$ will be defined turns out to be precisely the interval $[1, \delta]$. The sign of $r$ will be chosen depending on the value of $q$ and on whether we consider $\mathbf{B}_{\max }$ or $\mathbf{B}_{\min }$. Note that $y=1$ if $r=s=u_{p}(1 / \delta)$.

Now, we rewrite the equation for $g$ in terms of $r$ :

$$
\begin{aligned}
d(\log g) & =h d(\log y)=\frac{q(\gamma-1)}{1-\gamma r}\left(\frac{1}{1-r / p}-\frac{1}{1-r}\right) d r \\
& =\left(\frac{q}{1-r}-\frac{q-1}{p-r}-\frac{\gamma}{1-\gamma r}\right) d r
\end{aligned}
$$

With the initial condition $\left.g\right|_{y=1}=\left.g\right|_{r=s}=1$, we get

$$
\begin{aligned}
\log g & =\int_{r}^{s}\left(\frac{q}{1-t}-\frac{q-1}{p-t}-\frac{\gamma}{1-\gamma t}\right) d t \\
& =q \log \frac{1-s}{1-r}+(q-1) \log \frac{p-r}{p-s}+\log \frac{1-\gamma r}{1-\gamma s}
\end{aligned}
$$

i.e.,

$$
g(x ; p, q, \delta)=\left(\frac{1-s}{1-r}\right)^{q}\left(\frac{p-r}{p-s}\right)^{q-1} \frac{1-\gamma r}{1-\gamma s} .
$$

So, we arrive at the expression for the Bellman function $\mathbf{B}$ as in (16). It only remains to choose the signs of $r$ and $s$ so as to ensure (48).

After differentiating (49), we see that the derivative

$$
\frac{\partial h}{\partial r}=\frac{q \gamma(\gamma-1)}{(1-\gamma r)^{2}}
$$

does not change its sign. For $\mathbf{B}_{\max }$ condition (34) can be rewritten as $h(r) \geq h(0)$; for $\mathbf{B}_{\min }$ the reverse inequality comes into play. Therefore, for $\mathbf{B}_{\max }$ the sign of $\frac{\partial h}{\partial r}$ (i.e., the sign of $q \gamma(\gamma-1))$ coincides with the sign of $r$. Thus, $r=r^{+}$for $q \in\left[1-p^{\prime}, 0\right] \cup[1, \infty)$ and $r=r^{-}$for $q \in\left(-\infty, 1-p^{\prime}\right] \cup[0,1]$. Accordingly, for $\mathbf{B}_{\min }$ we must choose the opposite sign for $r$.

Note that, for $y$ to run over the interval $[1, \delta]$, the variable $r^{+}$must run over $\left[0, s^{+}\right]$or $r^{-}$must run over $\left[s^{-}, 0\right]$. Condition (48) imposed on $h$ allows $r$ to vary in the interval $(-\infty, 1 / \gamma)$ for positive $\gamma$ or in $(1 / \gamma, \infty)$ for negative $\gamma$; hence, $r^{+}$can run over the entire interval $\left[0, s^{+}\right]$only for $\gamma<1 / s^{+}$(i.e., for $q<\frac{p-s^{+}}{(p-1) s^{+}}$), and $r^{-}$can run over the entire interval $\left[s^{-}, 0\right]$ only for $\gamma>1 / s^{-}$(i.e., for $q>\frac{p-s^{-}}{(p-1) s^{-}}$). Therefore, we can use the variable $r^{+}$only when $\gamma<1 / s^{+}$and the variable $r^{-}$only when $\gamma>1 / s^{-}$. We arrive at the necessary condition $1 / s^{-}<\gamma<1 / s^{+}$or $\frac{p-s^{-}}{(p-1) s^{-}}<q<\frac{p-s^{+}}{(p-1) s^{+}}$for the function $y \mapsto h_{\max }(y)$ to be well defined for $y \in[1, \delta]$. But we already know that, for $q$ (or $\gamma$ ) outside of the intervals mentioned, the function $\mathbf{B}_{\max }(x ; p, q, \delta)$ is infinite everywhere except for the curve $x_{1} x_{2}^{p-1}=1$.

Thus, we have constructed the Bellman function as in Theorem 2 for $1<p<\infty$. An easy way to visualize the Bellman function for $p=\infty$ in Theorem $2_{\infty}$ is to pass to the limit as $p \rightarrow \infty$ in (13)-(14) taking into account that the variable $x_{1}$ should stay as it is, but $x_{2}^{p-1}$ should be replaced by $e^{-x_{2}}$ (i.e., $x_{2} \rightarrow 0$ ). This means that we simply replace $r_{p}^{ \pm}=u_{p}^{ \pm}\left(x_{1} x_{2}^{p-1}\right)$ by $r_{\infty}^{ \pm}=u_{\infty}^{ \pm}\left(x_{1} e^{-x_{2}}\right)$ and, therefore, $s_{p}^{ \pm}$by $s_{\infty}^{ \pm}$. Since $\gamma \rightarrow q$ as $p \rightarrow \infty$, we pass from (13)-(14) to $(36)-(37)$.

A more rigorous way to get these expressions is to repeat the procedure of finding an appropriate solution of the Bellman equation, as it was done for $1<p<\infty$. As before, 
we look for a concave function $\mathbf{B}_{\max }$ and a convex function $\mathbf{B}_{\min }$ on some domain $\Omega_{\delta}$. Now, the function $\mathbf{B}$ has the form

$$
\mathbf{B}\left(x_{1}, x_{2} ; q, \delta\right)=x_{1}^{q} g\left(x_{1} e^{-x_{2}} ; q, \delta\right),
$$

where $g(\cdot ; q, \delta)$ is a function on $[1, \delta]$ such that $g \geq 1$ for $q \notin(0,1), 0 \leq g \leq 1$ for $q \in[0,1]$, and the boundary condition $g(1 ; q, \delta)=1$ is fulfilled. All this is a consequence of the relation

$$
\mathbf{B}\left(t x_{1}, x_{2}+\log t ; q, \delta\right)=t^{q} \mathbf{B}\left(x_{1}, x_{2} ; q, \delta\right),
$$

which follows from the simultaneous consideration of two families of weights: $w, w \in$ $A_{\infty}^{\delta}(J)$, and $\tilde{w}=t w$, where $t$ is an arbitrary positive constant. The boundary condition $g(1 ; q, \delta)=1$ follows from the fact that the relation $x_{1}=e^{x_{2}}$ occurs if and only if $w=x_{1}=$ const, i.e., $\mathbf{B}\left(x_{1}, \log x_{1} ; q, \delta\right)=x_{1}^{q}$.

Again we introduce the new variable $y=x_{1} e^{-x_{2}}$ and calculate the matrix (32) by using the representation (51):

$$
\begin{aligned}
\frac{\partial y}{\partial x_{1}} & =\frac{y}{x_{1}}, \quad \frac{\partial y}{\partial x_{2}}=-y \\
\frac{\partial \mathbf{B}}{\partial x_{1}} & =q x_{1}^{q-1} g+x_{1}^{q} g^{\prime} \frac{\partial y}{\partial x_{1}}=x_{1}^{q-1}\left(q g+y g^{\prime}\right) \\
\frac{\partial \mathbf{B}}{\partial x_{2}} & =x_{1}^{q} g^{\prime} \frac{\partial y}{\partial x_{2}}=-x_{1}^{q} y g^{\prime} \\
\frac{\partial^{2} \mathbf{B}}{\partial x_{1}^{2}} & =(q-1) x_{1}^{q-2}\left(q g+y g^{\prime}\right)+x_{1}^{q-1}\left(q g^{\prime}+g^{\prime}+y g^{\prime \prime}\right) \frac{\partial y}{\partial x_{1}} \\
& =x_{1}^{q-2}\left(q(q-1) g+2 q y g^{\prime}+y^{2} g^{\prime \prime}\right) \\
\frac{\partial^{2} \mathbf{B}}{\partial x_{1} \partial x_{2}} & =-q x_{1}^{q-1} y g^{\prime}-x_{1}^{q}\left(g^{\prime}+y g^{\prime \prime}\right) \frac{\partial y}{\partial x_{1}}=-x_{1}^{q-1}\left((q+1) y g^{\prime}+y^{2} g^{\prime \prime}\right) \\
\frac{\partial^{2} \mathbf{B}}{\partial x_{2}^{2}} & =-x_{1}^{q}\left(y g^{\prime}\right)^{\prime} \frac{\partial y}{\partial x_{2}}=x_{1}^{q}\left(y g^{\prime}+y^{2} g^{\prime \prime}\right) .
\end{aligned}
$$

The matrix (32) turns into

$$
\left(\begin{array}{cc}
\frac{\partial^{2} \mathbf{B}}{\partial x_{1}^{2}} & \frac{\partial^{2} \mathbf{B}}{\partial x_{1} \partial x_{2}} \\
\frac{\partial^{2} \mathbf{B}}{\partial x_{1} \partial x_{2}} & \frac{\partial^{2} \mathbf{B}}{\partial x_{2}^{2}}
\end{array}\right)=x_{1}^{q-2}\left(\begin{array}{cc}
1 & -1 \\
0 & x_{1}
\end{array}\right) R\left(\begin{array}{cc}
1 & 0 \\
-1 & x_{1}
\end{array}\right),
$$

where

$$
R=\left(\begin{array}{cc}
q(q-1) g-y g^{\prime} & -q y g^{\prime} \\
-q y g^{\prime} & y g^{\prime}+y^{2} g^{\prime \prime}
\end{array}\right) .
$$

As before, we require that this matrix be singular:

$$
\left(q(q-1) g-y g^{\prime}\right)\left(y g^{\prime}+y^{2} g^{\prime \prime}\right)=\left(q y g^{\prime}\right)^{2},
$$

and negative definite (for $\mathbf{B}_{\max }$ ) or positive definite (for $\mathbf{B}_{\min }$ ):

$$
y g_{\min }^{\prime}-q(q-1) g_{\min } \leq 0 \leq y g_{\max }^{\prime}-q(q-1) g_{\max } .
$$

It is natural that (53) and (54) are the limit cases of (45) and (46) as $p \rightarrow \infty$.

As before, we make the substitution $h=y g^{\prime} / g$ and solve equation (53):

$$
y=y_{\infty} \frac{h+q}{h} \exp \left(-\frac{q-1}{h}\right) ;
$$

inequality (54) turns into

$$
h_{\min }-q(q-1) \leq 0 \leq h_{\max }-q(q-1)
$$


Again we introduce a new variable $r$ by putting

$$
r=\frac{h-q(q-1)}{q h} \text { or } h=\frac{q(q-1)}{1-q r} .
$$

Then (55) turns into

$$
y=\delta(1-r) e^{r}
$$

where $\delta=y_{\infty} \frac{q}{q-1} \exp (-1 / q)$, so that $r=r^{ \pm}=u^{ \pm}(y / \delta)$. As before, the sign of $r$ is determined by (56). Finally, we have

$$
\mathbf{B}(x ; q, \delta)=x_{1}^{q} g(y ; q, \delta)=x_{1}^{q} \frac{1-q r}{1-q s}\left(\frac{1-r}{1-s}\right)^{q} .
$$

Now, we pass to the more difficult limit case of $p=1$. Of course, to find a candidate for the Bellman function we can pass to the limit as $p \rightarrow 1$ in formulas (13)-(14). How to do this was explained immediately before the statement of Theorem $2_{1}$. However, we try to adapt the arguments used above to the particular case in question. The situation is noteworthy, because condition (38) is no longer the usual concavity, and (39) is not the usual convexity.

As before, we consider three points in $\Omega_{\delta}(1): x^{ \pm}$and $x^{0}=\left(\alpha^{-} x_{1}^{-}+\alpha^{+} x_{1}^{+}, \max x_{2}^{ \pm}\right)$. For a given weight $w$, we get such a triple if we take $x^{0}=\left(\langle w\rangle_{I}, \sup _{I} 1 / w\right), x^{ \pm}=$ $\left(\langle w\rangle_{I^{ \pm}}, \sup _{I^{ \pm}} 1 / w\right)$, where $I=I^{-} \cup I^{+}$is a splitting with $\left|I^{ \pm}\right|=\alpha^{ \pm}|I|$.

Assuming that the function $\mathbf{B}$ is sufficiently smooth, we take its Taylor expansion near the point $x^{0}$ up to the second order terms:

$$
\mathbf{B}\left(x^{ \pm}\right) \approx \mathbf{B}\left(x^{0}\right)+\sum_{i=1}^{2} \frac{\partial \mathbf{B}}{\partial x_{i}}\left(x^{0}\right)\left(x_{i}^{ \pm}-x_{i}^{0}\right)+\frac{1}{2} \sum_{i, j=1}^{2} \frac{\partial^{2} \mathbf{B}}{\partial x_{i} \partial x_{j}}\left(x^{0}\right)\left(x_{i}^{ \pm}-x_{i}^{0}\right)\left(x_{j}^{ \pm}-x_{j}^{0}\right) .
$$

Since one of the values $x_{2}^{ \pm}$coincides with $x_{2}^{0}$, for definiteness we assume that $x_{2}^{+}=x_{2}^{0}$ and $\Delta_{2} \stackrel{\text { def }}{=} x_{2}^{0}-x_{2}^{-} \geq 0$. Recalling that $x_{1}^{0}=\alpha^{-} x_{1}^{-}+\alpha^{+} x_{1}^{+}$, we put $\Delta_{1}=x_{1}^{+}-x_{1}^{-}$. Then, taking the linear combination of the expressions (57) with the coefficients $\alpha^{ \pm}$, we get

$$
\begin{aligned}
& \alpha^{-} \mathbf{B}\left(x^{-}\right)+\alpha^{+} \mathbf{B}\left(x^{+}\right)-\mathbf{B}\left(x^{0}\right) \\
& \quad \approx-\alpha^{-} \frac{\partial \mathbf{B}}{\partial x_{2}} \Delta_{2}+\frac{1}{2} \alpha^{-} \alpha^{+} \frac{\partial^{2} \mathbf{B}}{\partial x_{1}^{2}} \Delta_{1}^{2}+\alpha^{-} \alpha^{+} \frac{\partial^{2} \mathbf{B}}{\partial x_{1} \partial x_{2}} \Delta_{1} \Delta_{2}+\frac{1}{2} \alpha^{-} \frac{\partial^{2} \mathbf{B}}{\partial x_{2}} \Delta_{2}^{2} .
\end{aligned}
$$

For $\mathbf{B}_{\max }$ this expression must be nonpositive for all small $\Delta_{1}$ and small positive $\Delta_{2}$. This yields

$$
\frac{\partial \mathbf{B}_{\max }}{\partial x_{2}} \geq 0, \quad \frac{\partial^{2} \mathbf{B}_{\max }}{\partial x_{1}^{2}} \leq 0 .
$$

For $\mathbf{B}_{\text {min }}$ the reverse inequalities must be true. Supplementing this by the singularity condition (which just provides the Bellman equation, as a matter of fact), we get two possibilities:

$$
\begin{array}{rlrl}
\frac{\partial^{2} \mathbf{B}}{\partial x_{1}^{2}} & =0, & \frac{\partial \mathbf{B}_{\max }}{\partial x_{2}} \geq 0, & \frac{\partial \mathbf{B}_{\min }}{\partial x_{2}} \leq 0 ; \\
\frac{\partial \mathbf{B}}{\partial x_{2}}=0, & \frac{\partial^{2} \mathbf{B}_{\max }}{\partial x_{1}^{2}} \leq 0, & \frac{\partial^{2} \mathbf{B}_{\min }}{\partial x_{1}^{2}} \geq 0 .
\end{array}
$$

Together with a weight $w \in A_{1}^{\delta}$ we consider the family of weights $\tilde{w}=t w$; this yields the identity

$$
\mathbf{B}\left(t x_{1}, \frac{x_{2}}{t} ; p, q, \delta\right)=t^{q} \mathbf{B}\left(x_{1}, x_{2} ; p, q, \delta\right), \quad t>0
$$


Putting $t=x_{2}$ we obtain the relation

$$
\mathbf{B}\left(x_{1} x_{2}, 1 ; p, q, \delta\right)=x_{2}^{q} \mathbf{B}\left(x_{1}, x_{2} ; p, q, \delta\right),
$$

whence $\mathbf{B}(x)=x_{1}^{q} g\left(x_{1} x_{2}\right)$, where $g$ is a function on $[1, \delta]$ defined by the formula $g(y)=$ $y^{-q} \mathbf{B}(y, 1)$.

As before, by the Hölder inequality (see (41)) we have $\mathbf{B}(x) \geq x_{1}^{q}$ for $q \notin(0,1)$ and $\mathbf{B}(x) \leq x_{1}^{q}$ for $q \in[0,1]$, which implies that $g \geq 1$ for $q \notin(0,1)$ and $0 \leq g \leq 1$ for $q \in[0,1]$. Moreover, since the relation $x_{1} x_{2}=1$ occurs if and only if $w=x_{1}=$ const, we have $\mathbf{B}\left(x_{1}, x_{1}^{-1} ; q, \delta\right)=x_{1}^{q}$, i.e., $g(1 ; q, \delta)=1$.

First we find a solution satisfying (58). This must be a linear function in $x_{1}$, i.e.,

$$
\mathbf{B}(x)=a\left(x_{2}\right)+b\left(x_{2}\right) x_{1}=x_{1}^{q} g\left(x_{1} x_{2}\right) .
$$

Putting $x_{1}=x_{2}^{-1}$, we get $a\left(x_{2}\right)=x_{2}^{-q}-b\left(x_{2}\right) x_{2}^{-1}$, whence

$$
x_{2}^{-q}+b\left(x_{2}\right)\left(x_{1} x_{2}-1\right) x_{2}^{-1}=x_{1}^{q} g\left(x_{1} x_{2}\right) .
$$

Therefore,

and

$$
b\left(x_{2}\right) x_{2}^{q-1}=\frac{\left(x_{1} x_{2}\right)^{q} g\left(x_{1} x_{2}\right)-1}{x_{1} x_{2}-1} \stackrel{\text { def }}{=} c=\text { const },
$$

$$
\begin{aligned}
\mathbf{B}(x) & =c x_{1} x_{2}^{1-q}+(1-c) x_{2}^{-q}, \\
g(y) & =c y^{1-q}+(1-c) y^{-q} .
\end{aligned}
$$

Since the function $y \mapsto \frac{y^{q}-1}{y-1}$ increases monotonically for $q \notin(0,1)$ and decreases monotonically for $q \in[0,1]$, and

$$
g(y)-1=y^{-q}(y-1)\left(c-\frac{y^{q}-1}{y-1}\right)
$$

the constant $c$ satisfies the condition

$$
\begin{aligned}
& c \geq \frac{\delta^{q}-1}{\delta-1}>q \quad \text { for } q \notin[0,1], \\
& c \leq \frac{\delta^{q}-1}{\delta-1}<q \quad \text { for } q \in(0,1) .
\end{aligned}
$$

Now we examine the sign of the derivative

$$
\frac{\partial \mathbf{B}}{\partial x_{2}}=c(1-q) x_{1} x_{2}^{-q}-(1-c) q x_{2}^{-q-1}=x_{2}^{-q-1}\left[c(1-q) x_{1} x_{2}-(1-c) q\right] .
$$

The linear function $\varphi: y \mapsto c(1-q) y-(1-c) q$ must keep its sign on the interval $y \in[1, \delta]$. But we know its sign at the point $y=1$, because by (60) we have $c-q>0$ for $q \notin[0,1]$ and $c-q<0$ for $q \in(0,1)$. Therefore, this function is a candidate for $\mathbf{B}_{\max }$ if $q \notin[0,1]$ and for $\mathbf{B}_{\min }$ if $q \in(0,1)$. Note that there is no admissible value of $c$ for $q \geq \frac{\delta}{\delta-1}$, because then $(1-q) \delta+q \leq 0$ and the restriction $\varphi(\delta)=c[(1-q) \delta+q]-q \geq 0$ contradicts condition (60). For the other $q$ it is natural to choose $c$ in such a way that $[1, \delta]$ be the maximum interval where the function $\varphi$ does not change its sign, i.e., to solve the equation $\delta=\frac{(1-c) q}{c(1-q)}$, whence

$$
c=\frac{q}{q+(1-q) \delta},
$$

and for $\mathbf{B}$ we get the expression written in formulas (36)-(37).

Now we consider the second case, where conditions (59) are fulfilled. This implies immediately that in the representation $\mathbf{B}=x_{1}^{q} g\left(x_{1} x_{2}\right)$ the function $g$ is constant and, 
consequently, $g=1$, i.e., $\mathbf{B}=x_{1}^{q}$. To understand which of the two functions $\mathbf{B}_{\min }$ or $\mathbf{B}_{\max }$ it represents, we need only to find the sign of its second derivative. Since

$$
\frac{\partial^{2} \mathbf{B}}{\partial x_{1}^{2}}=q(q-1) x_{1}^{q-2},
$$

we have $\mathbf{B}_{\min }=x_{1}^{q}$ for $q \notin(0,1)$ and $\mathbf{B}_{\max }=x_{1}^{q}$ for $q \in[0,1]$. This yields the second half of formulas (36)-(37).

Appendix 2: how to find an extremal function. In the proof of Lemma 1 the required extremal function appeared without any explanations. Now we explain how to find it.

Since for an extremal function we have equality in (26) on the corresponding side (of course, each of $\mathbf{B}_{\min }$ and $\mathbf{B}_{\max }$ has its own extremal function), we need to have equality on each splitting step in the chain of inequalities (27). This means that in the splitting process we "go" only along the vector field determined by the kernel vectors of the matrix (23). From (24) we get the equation for the trajectories on which the functions $\mathbf{B}$ are linear:

$$
x_{2}(1-r) d x_{1}+(p-1) x_{1} d x_{2}=0 .
$$

The corresponding trajectories are the family of the segments of the tangent lines

$$
b^{p} x_{1}+\delta(p-1) x_{2}=\delta b p
$$

to the graph $x_{1} x_{2}^{p-1}=\delta$ between the points $\left(\delta p b^{1-p} \frac{1-s}{p-s}, \frac{b p}{p-s}\right)$ and $\left(\delta b^{1-p}, b\right)$. We check this claim.

Together with (61), we use the definition of $r: r=u_{p}^{ \pm}\left(\frac{1}{\delta} x_{1} x_{2}^{p-1}\right)$, i.e.,

$$
\delta(1-r)\left(1-\frac{r}{p}\right)^{-p}=x_{1} x_{2}^{p-1},
$$

whence

$$
-\frac{(p-1) r d r}{(1-r)(p-r)}=\frac{d x_{1}}{x_{1}}+(p-1) \frac{d x_{2}}{x_{2}} .
$$

Combined with (61), this equation yields

$$
x_{1}(r)=x_{1}(0) p \frac{1-r}{p-r}, \quad x_{2}(r)=\frac{x_{2}(0) p}{p-r} .
$$

If we take $x_{2}(0)=b$ as a free parameter, then from (63) with $r=0$ we get $x_{1}(0)=\delta b^{1-p}$, and excluding $r$ from (64) we obtain equation (62). In fact, we have got two families of straight segments:

$$
\begin{aligned}
& \omega_{\delta}^{+}(b)=\left\{x=\left(\delta p b^{1-p} \frac{1-r}{p-r}, \frac{b p}{p-r}\right): 0 \leq r \leq s^{+}\right\}, \\
& \omega_{\delta}^{-}(b)=\left\{x=\left(\delta p b^{1-p} \frac{1-r}{p-r}, \frac{b p}{p-r}\right): s^{-} \leq r \leq 0\right\} .
\end{aligned}
$$

Each of them covers the entire domain $\Omega_{\delta}$ :

$$
\Omega_{\delta}=\bigcup_{b>0} \omega_{\delta}^{+}(b)=\bigcup_{b>0} \omega_{\delta}^{-}(b) .
$$

The functions $\mathbf{B}_{\max }$ and $\mathbf{B}_{\min }$ are linear on the corresponding segments (we must take the same sign for $\omega$ that is taken for $r$ in the expression for $\mathbf{B}$ in accordance with 
formulas (13)-(14)). Indeed,

$$
\begin{aligned}
x_{1}^{\gamma} x_{2}^{1-\gamma} & \left(\frac{1-s}{1-r}\right)^{\gamma} \frac{1-\gamma r}{1-\gamma s}=\left(\delta p b^{1-p} \frac{1-r}{p-r}\right)^{\gamma}\left(\frac{b p}{p-r}\right)^{1-\gamma}\left(\frac{1-s}{1-r}\right)^{\gamma} \frac{1-\gamma r}{1-\gamma s} \\
= & \delta^{\gamma} p b^{1-p \gamma} \frac{(1-s)^{\gamma}}{1-\gamma s} \frac{1-\gamma r}{p-r} \\
& =\delta^{\gamma} b^{-p \gamma} \frac{(1-s)^{\gamma}}{1-\gamma s}\left(b p \gamma+(1-\gamma p) x_{2}\right) .
\end{aligned}
$$

Note that we have yet another "admissible trajectory", which is the envelope of the segments $\omega_{\delta}^{+}(b)\left(\right.$ or $\left.\omega_{\delta}^{-}(b)\right): x_{1} x_{2}^{p-1}=\delta$.

First we consider the case of points on this critical line: $x_{2}^{0}$ is an arbitrary positive number and $x_{1}^{0}=\delta\left(x_{2}^{0}\right)^{1-p}$. Let $I=[0,1]$. We split this interval at a point $a: I^{-}=[0, a]$, $I^{+}=[a, 1]$. The points $x^{ \pm}$must be on our "admissible trajectory", i.e., on the graph $x_{1} x_{2}^{p-1}=\delta$ on one side of $x^{0}$, and on the straight line $x_{2}^{0} x_{1}+(p-1) x_{1}^{0} x_{2}=p x_{1}^{0} x_{2}^{0}$ on the other side. By symmetry, we may assume that $x^{-}$is on the graph $x_{1} x_{2}^{p-1}=\delta$. Then for all $a \in[0,1]$ we have

$$
\left(\frac{1}{a} \int_{0}^{a} w(t) d t\right)\left(\frac{1}{a} \int_{0}^{a} w(t)^{1-p^{\prime}} d t\right)^{p-1}=\delta .
$$

If we introduce

$$
v(a)=\int_{0}^{a} w(t)^{1-p^{\prime}} d t
$$

then, substituting $\int_{0}^{a} w(t)^{1-p^{\prime}} d t=v$ in (65), we get

$$
\begin{gathered}
\left(\frac{1}{a} \int_{0}^{a} v^{\prime}(t)^{1-p} d t\right)\left(\frac{v}{a}\right)^{p-1}=\delta, \\
\int_{0}^{a} v^{\prime}(t)^{1-p} d t=\delta a^{p} v^{1-p} \\
\left(v^{\prime}\right)^{1-p}=\delta p t^{p-1} v^{1-p}+\delta t^{p}(1-p) v^{-p} v^{\prime}, \\
1=\delta\left(p-(p-1) \frac{t v^{\prime}}{v}\right)\left(\frac{t v^{\prime}}{v}\right)^{p-1}, \\
(1-s)\left(1-\frac{s}{p}\right)^{-p}=\frac{1}{\delta}=\left[1-p\left(1-\frac{v}{t v^{\prime}}\right)\right]\left(\frac{v}{t v^{\prime}}\right)^{-p} .
\end{gathered}
$$

Therefore,

$$
\begin{gathered}
p\left(1-\frac{v}{t v^{\prime}}\right)=s, \\
\frac{d v}{v}=\frac{p}{p-s} \frac{d t}{t}, \\
v=\text { const } \cdot \frac{p}{p-s}, \\
w^{1-p^{\prime}}=v^{\prime}=\text { const } \cdot t^{\frac{s}{p-s}}, \\
w=\text { const } \cdot t^{\frac{s(1-p)}{p-s}} .
\end{gathered}
$$

To normalize the solution by the condition $\langle w\rangle_{I}=x_{1}^{0}$, we must take const $=x_{1}^{0} \frac{p(1-s)}{p-s}$, and finally we obtain

$$
w(t)=x_{1}^{0} \frac{p(1-s)}{p-s} t^{\frac{s(1-p)}{p-s}}
$$


Note that $\frac{s(1-p)}{p-s}>-1$ (since $s<1$ ), whence $w \in L^{1}(I)$. We check that also $w^{1-p^{\prime}} \in$ $L^{1}(I)$ and $x_{1}^{0}\left(x_{2}^{0}\right)^{p-1}=\delta$. Since

$$
\frac{s(1-p)}{p-s} \cdot\left(1-p^{\prime}\right)=\frac{s}{p-s}>-1
$$

the function $w^{1-p^{\prime}}$ is integrable, and

$$
\begin{aligned}
x_{2}^{0} & =\left\langle w^{1-p^{\prime}}\right\rangle=\left(x_{1}^{0} \frac{p(1-s)}{p-s}\right)^{1-p^{\prime}} \int_{0}^{1} t^{\frac{s}{p-s}} d t=\left(x_{1}^{0}(1-s)\right)^{1-p^{\prime}}\left(\frac{p-s}{p}\right)^{p^{\prime}}, \\
x_{1}^{0}\left(x_{2}^{0}\right)^{p-1} & =x_{1}^{0}\left(x_{1}^{0}(1-s)\right)^{-1}\left(\frac{p-s}{p}\right)^{p^{\prime}(p-1)}=\frac{1}{1-s}\left(1-\frac{s}{p}\right)^{p}=\delta .
\end{aligned}
$$

Now, let $x^{0}$ be an arbitrary point in $\Omega_{\delta}$. Then we make the following splitting: $x^{-}=\left(\delta\left(x_{2}^{-}\right)^{1-p}, x_{2}^{-}\right)$and $x^{+}=\left(\left(x_{2}^{+}\right)^{1-p}, x_{2}^{+}\right)$, where both points $x^{ \pm}$are on the line $(62)$ with $b=x_{2}^{-}$, i.e., on the tangent line to the graph $x_{1} x_{2}^{p-1}=\delta$ at the point $x^{-}$, to guarantee equality in (18) (or in (19)). Since the point $x^{+}$corresponds to the value $r=s$ in (64), and the point $x^{0}$ corresponds to the value $r=r^{0}=u_{p}\left(\frac{1}{\delta} x_{1}^{0}\left(x_{2}^{0}\right)^{p-1}\right)$, equations (64) yield

$$
\begin{array}{ll}
x_{1}^{0}=x_{1}^{-} p \frac{1-r^{0}}{p-r^{0}}, & x_{1}^{+}=x_{1}^{-} p \frac{1-s}{p-s}, \\
x_{2}^{0}=\frac{p x_{2}^{-}}{p-r^{0}}, & x_{2}^{+}=\frac{p x_{2}^{-}}{p-s} .
\end{array}
$$

For the splitting point $a=\alpha^{-}$we have

$$
x_{2}^{0}-x_{2}^{+}=\alpha^{-}\left(x_{2}^{-}-x_{2}^{+}\right) \text {, }
$$

whence

$$
a=\alpha^{-}=\frac{p\left(s-r^{0}\right)}{s\left(p-r^{0}\right)} .
$$

Since $x_{1}^{+}\left(x_{2}^{+}\right)^{p-1}=1$, the function $w$ is constant on $I^{+}$, i.e.,

$$
\left.w\right|_{I^{+}}=x_{1}^{+}=x_{1}^{0} \frac{(1-s)\left(p-r^{0}\right)}{\left(1-r^{0}\right)(p-s)},
$$

and for the interval $I^{-}$we must take the extremal function (66) renormed to this interval:

$$
\left.w\right|_{I^{-}}=x_{1}^{-} \frac{p(1-s)}{p-s}\left(\frac{t}{a}\right)^{\frac{s(1-p)}{p-s}}=x_{1}^{0} \frac{(1-s)\left(p-r^{0}\right)}{\left(1-r^{0}\right)(p-s)}\left(\frac{t}{a}\right)^{\frac{s(1-p)}{p-s}} .
$$

This yields the extremal function we used in the proof of Lemma 1.

Passing to the limit as $p \rightarrow \infty$, we get the extremal function

$$
w(t)= \begin{cases}x_{1}^{+}\left(\frac{a}{t}\right)^{s} & \text { if } 0 \leq t \leq a, \\ x_{1}^{+} & \text {if } a \leq t \leq 1\end{cases}
$$

that was used in the proof of Lemma $1_{\infty}$.

Of course, this extremal function can be obtained by repeating the construction for $p<\infty$, which clarifies the situation to a greater extent than a formal limit passage. The trajectories where the functions $\mathbf{B}$ are linear (i.e., the vector field given by the kernel of $(32))$ are

$$
\begin{aligned}
& \omega_{\delta}^{+}(b)=\left\{x=\left(\delta e^{b}(1-r), b-r\right): 0 \leq r \leq s^{+}\right\} \\
& \omega_{\delta}^{-}(b)=\left\{x=\left(\delta e^{b}(1-r), b-r\right): s^{-} \leq r \leq 0\right\}
\end{aligned}
$$


They are the segments of the tangent line

$$
x_{1}=\delta e^{b}\left(x_{2}-b+1\right)
$$

to the graph $x_{1}=\delta e^{x_{2}}$ between the points $\left(e^{b-s}, b-s\right)$ and $\left(\delta e^{b}, b\right)$.

After the above analysis it is useful to repeat the remark made before the statement of Theorem $2_{\infty}$ : it would be more natural to consider the variable $x_{2}=-(p-1) \log \left\langle w^{\frac{1}{1-p}}\right\rangle_{I}$ rather than $x_{2}=\left\langle w^{\frac{1}{1-p}}\right\rangle_{I}$. But this becomes clear a posteriori, when we have found and investigated the Bellman function for various $p$. We review briefly the changes that happen if we take $x_{2}=-(p-1) \log \left\langle w^{\frac{1}{1-p}}\right\rangle_{I}$ as the second variable. For such a choice of $x_{2}$ the domain $\Omega_{\delta}(p)$ is the same for all $p$, namely,

$$
\Omega_{\delta}=\left\{\left(x_{1}, x_{2}\right): x_{2} \in \mathbb{R}, 1 \leq x_{1} e^{-x_{2}} \leq \delta\right\} .
$$

For all $p$ the Bellman function has the form

$$
\mathbf{B}(x ; p, q, \delta)=x_{1}^{q} g(y ; p, q, \delta)
$$

with $y=x_{1} e^{-x_{2}}$. Now, in Lemma 3 we must consider "generalized" convexity/concavity:

$$
\begin{gathered}
B_{\max }\left(x^{0}\right) \geq \alpha^{-} B_{\max }\left(x^{-}\right)+\alpha^{+} B_{\max }\left(x^{+}\right), \\
B_{\min }\left(x^{0}\right) \leq \alpha^{-} B_{\min }\left(x^{-}\right)+\alpha^{+} B_{\min }\left(x^{+}\right),
\end{gathered}
$$

where

$$
\begin{aligned}
& x_{1}^{0}=\alpha^{-} x_{1}^{-}+\alpha^{+} x_{1}^{+}, \\
& x_{2}^{0}=-(p-1) \log \left(\alpha^{-} e^{-\frac{x_{2}^{-}}{p-1}}+\alpha^{+} e^{-\frac{x_{2}^{+}}{p-1}}\right),
\end{aligned}
$$

i.e., the three points $x^{ \pm}, x^{0}$ are on the curve

$$
x_{2}=-(p-1) \log \left(a x_{1}+b\right),
$$

which now plays the role of a straight line. Any two points $x^{ \pm}$can be joined by a unique curve of this form with the parameters

$$
a=\frac{e^{-\frac{x_{2}^{+}}{p-1}}-e^{-\frac{x_{2}^{-}}{p-1}}}{x_{1}^{+}-x_{1}^{-}}, \quad b=\frac{x_{1}^{+} e^{-\frac{x_{2}^{+}}{p-1}}-x_{1}^{-} e^{-\frac{x_{2}^{-}}{p-1}}}{x_{1}^{+}-x_{1}^{-}},
$$

and the point $x^{0}$ given by (68) is on the same curve. The concavity/convexity condition (67) is concavity/convexity along the lines of the form (69). Surely, the quadratic form responsible for the corresponding property changes. Now, the role of the matrix in (23) will be played by the matrix

$$
\left(\begin{array}{cc}
\frac{\partial^{2} B}{\partial x_{1}^{2}} & \frac{\partial^{2} B}{\partial x_{1} \partial x_{2}} \\
\frac{\partial^{2} B}{\partial x_{1} \partial x_{2}} & \frac{\partial^{2} B}{\partial x_{2}^{2}}+\frac{1}{p-1} \cdot \frac{\partial B}{\partial x_{2}}
\end{array}\right) .
$$

Calculating the quadratic form of this matrix, we get

$$
-\frac{\gamma q(q-1) x_{1}^{q-2}}{r(1-\gamma s)}\left(\frac{1-s}{1-r}\right)^{q}\left(\frac{p-r}{p-s}\right)^{q-1}\left((1-r) \Delta_{1}-x_{1} \Delta_{2}\right)^{2} .
$$

Now, the trajectories $\omega^{ \pm}$determined by the kernel of the matrix (70) are the pieces of a logarithmic curve that touches the graph $x_{2}=\log \frac{x_{1}}{\delta}$. If the tangency point is denoted by $\kappa=\left(\kappa_{1}, \kappa_{2}\right)\left(\kappa_{1} e^{-\kappa_{2}}=\delta\right)$, then

$$
\begin{aligned}
& \omega_{\delta}^{+}(\kappa)=\left\{x=\left(\kappa_{1} \frac{p(1-r)}{p-r}, \kappa_{2}+(p-1) \log \frac{p-r}{p}\right): 0 \leq r \leq s^{+}\right\}, \\
& \omega_{\delta}^{-}(\kappa)=\left\{x=\left(\kappa_{1} \frac{p(1-r)}{p-r}, \kappa_{2}+(p-1) \log \frac{p-r}{p}\right): s^{-} \leq r \leq 0\right\} .
\end{aligned}
$$


In the limit case as $p \rightarrow \infty$, these segments of the logarithmic curve tend to segments of the straight line that touches the boundary of $\Omega_{\delta}$ at the point $\kappa$.

To consider the other limit case $(p \rightarrow 1)$, we need to renormalize the basis in which we calculate the matrix (70), namely, we divide the second vector by $\sqrt{p-1}$. Then we can pass to the limit

$$
\left(\begin{array}{cc}
\frac{\partial^{2} B}{\partial x_{1}^{2}} & \sqrt{p-1} \frac{\partial^{2} B}{\partial x_{1} \partial x_{2}} \\
\sqrt{p-1} \frac{\partial^{2} B}{\partial x_{1} \partial x_{2}} & (p-1) \frac{\partial^{2} B}{\partial x_{2}^{2}}+\frac{\partial B}{\partial x_{2}}
\end{array}\right) \longrightarrow\left(\begin{array}{cc}
\frac{\partial^{2} B}{\partial x_{1}^{2}} & 0 \\
0 & \frac{\partial B}{\partial x_{2}}
\end{array}\right) .
$$

Since the limit matrix is diagonal, the Bellman equation "factorizes" and we get two independent equations: $\frac{\partial^{2} B}{\partial x_{1}^{2}}=0$ and $\frac{\partial B}{\partial x_{2}}=0$.

In this limit the curve $\omega^{+}(\kappa)$ turns into a horizontal straight segment and $\omega^{-}(\kappa)$ into a vertical one:

$$
\begin{aligned}
& \omega_{\delta}^{+}(\kappa)=\left\{x=\left(\kappa_{1} t, \kappa_{2}\right): \frac{1}{\delta} \leq t \leq 1\right\}, \\
& \omega_{\delta}^{-}(\kappa)=\left\{x=\left(\kappa_{1}, \kappa_{2}+t\right): 0 \leq t \leq \log \delta\right\} .
\end{aligned}
$$

Appendix 3: how to calculate the " $A_{p}$-norm" of the extremal function. Since the " $A_{p}$-norm" (2) (or (1) for $p=\infty$ ) does not change after multiplication of the weight by a constant, we calculate the supremum of the expression

$$
\langle w\rangle_{I}\left\langle w^{1-p^{\prime}}\right\rangle_{I}^{p-1}
$$

for the weight

$$
w_{a, \nu}(t)= \begin{cases}a^{\nu} t^{-\nu} & \text { if } 0 \leq t \leq a \\ 1 & \text { if } a \leq t \leq 1\end{cases}
$$

We shall consider $I=[\alpha, \beta]$ with $0 \leq \alpha<a \leq \beta \leq 1$. The average $\langle w\rangle_{[0,1]}$ is finite if $\nu<1$, and $\left\langle w^{1-p^{\prime}}\right\rangle_{I}^{p-1}$ is finite if $\nu>1-p$; in what follows we assume that $1-p<\nu<1$. Direct calculation yields

$$
\begin{aligned}
\langle w\rangle_{I} & =\frac{\beta(1-\nu)+a \nu-\alpha^{1-\nu} a^{\nu}}{(1-\nu)(\beta-\alpha)} \\
\left\langle w^{1-p^{\prime}}\right\rangle_{I} & =\frac{\beta\left[1+\nu\left(p^{\prime}-1\right)\right]-a \nu\left(p^{\prime}-1\right)-\alpha^{1+\nu\left(p^{\prime}-1\right)} a^{-\nu\left(p^{\prime}-1\right)}}{\left[1+\nu\left(p^{\prime}-1\right)\right](\beta-\alpha)} .
\end{aligned}
$$

We introduce two new variables

$$
\lambda=\frac{\alpha^{1-\nu} a^{\nu}}{\beta(1-\nu)+a \nu}, \quad \mu=\frac{\alpha^{1+\nu\left(p^{\prime}-1\right)} a^{-\nu\left(p^{\prime}-1\right)}}{\beta\left[1+\nu\left(p^{\prime}-1\right)\right]-a \nu\left(p^{\prime}-1\right)} .
$$

The restriction $0 \leq \alpha<a \leq \beta$ implies $0 \leq \lambda<1,0 \leq \mu<1$. We introduce yet another parameter

$$
K=\left(1-\nu+\frac{a \nu}{\beta}\right)\left[1+\nu\left(p^{\prime}-1\right)-\frac{a \nu\left(p^{\prime}-1\right)}{\beta}\right]^{p-1}=(1-\tau)\left(1+\frac{\tau}{p-1}\right)^{p-1},
$$

where the variable $\tau=\nu(1-a / \beta)$ is in the same interval $1-p<\tau<1$ as $\nu$. Then $0<K \leq 1$. Indeed, the function $\tau \mapsto K(\tau)$ increases monotonically from 0 to 1 when $\tau$ runs from $1-p$ to 0 , and decreases monotonically from 1 to 0 when $\tau$ runs from 0 to 1 . Rewriting (71) in these new terms, we obtain

$$
\langle w\rangle_{I}\left\langle w^{1-p^{\prime}}\right\rangle_{I}^{p-1}=\frac{K(1-\lambda)(1-\mu)^{p-1}}{(1-\alpha / \beta)^{p}} \delta,
$$

where

$$
\delta=\frac{1}{(1-\nu)\left[1+\nu\left(p^{\prime}-1\right)\right]^{p-1}}
$$


We replace the fraction $\alpha / \beta$ in the denominator by $K^{1 / p} \lambda^{1 / p} \mu^{1 / p^{\prime}}$. We can do this because

$$
\lambda^{1 / p} \mu^{1 / p^{\prime}}=\frac{\alpha^{\frac{1-\nu}{p}} a^{\nu / p}}{(\beta(1-\nu)+a \nu)^{1 / p}} \frac{\alpha^{\frac{1+\nu\left(p^{\prime}-1\right)}{p^{\prime}}} a^{-\nu \frac{p^{\prime}-1}{p^{\prime}}}}{\left(\beta\left[1+\nu\left(p^{\prime}-1\right)\right]-a \nu\left(p^{\prime}-1\right)\right)^{1 / p^{\prime}}}=\frac{\alpha}{\beta K^{1 / p}} .
$$

Finally, we get

$$
\langle w\rangle_{I}\left\langle w^{1-p^{\prime}}\right\rangle_{I}^{p-1}=\delta \frac{K(1-\lambda)(1-\mu)^{p-1}}{\left(1-K^{1 / p} \lambda^{1 / p} \mu^{1 / p^{\prime}}\right)^{p}} .
$$

To show that the " $A_{p}$-norm" of $w_{a, \nu}$ is $\delta$, we need only to check that the above fraction does not exceed 1. Indeed, this fraction is equal to 1 for $\alpha=0$ and $\beta=a$, when $K=1$ and $\lambda=\mu=0$. Observe that the intervals with $\beta<a$ do not require any special treatment, because for such $\beta$ the expression (71) does not depend on $a$ ( $a^{\nu}$ is a multiplicative constant) and we can take the expression (72) with $a=\beta$.

The remaining arguments are formal and easy. We consider the function

$$
\varphi(\lambda, \mu)=\frac{(1-\lambda)(1-\mu)^{p-1}}{\left(1-K^{1 / p} \lambda^{1 / p} \mu^{1 / p^{\prime}}\right)^{p}}
$$

on the square $0 \leq \lambda \leq 1,0 \leq \mu \leq 1$. Since

$$
\frac{\partial \varphi}{\partial \lambda}=\frac{\lambda^{-1 / p^{\prime}}(1-\mu)^{p-1}\left(K^{1 / p} \mu^{1 / p^{\prime}}-\lambda^{1 / p^{\prime}}\right)}{\left(1-K^{1 / p} \lambda^{1 / p} \mu^{1 / p^{\prime}}\right)^{p}}
$$

$\varphi$ takes its maximum value for $\lambda=K^{p^{\prime}-1} \mu$ :

$$
\varphi\left(K^{p^{\prime}-1} \mu, \mu\right)=\left(\frac{1-\mu}{1-K^{p^{\prime}-1} \mu}\right)^{p-1} .
$$

Since the latter function is monotone decreasing in $\mu$, its maximum value is 1 for $\mu=0$, i.e.,

$$
\langle w\rangle_{I}\left\langle w^{1-p^{\prime}}\right\rangle_{I}^{p-1} \leq K \delta \leq \delta=\frac{1}{(1-\nu)\left[1+\nu\left(p^{\prime}-1\right)\right]^{p-1}} .
$$

Note that the " $A_{p}$-norm" of $w_{a, \nu}$ does not depend on $a$. So, fixing $\delta$ (i.e., $\nu$ ) and varying $c$ and $a$, we can get an example of a weight representing any point of the domain $\Omega_{\delta}$. If we need a function $w_{a, \nu}$ of a prescribed " $A_{p}$-norm" $\delta$, we must solve the equation

$$
\frac{1}{\delta}=(1-\nu)\left[1+\nu\left(p^{\prime}-1\right)\right]^{p-1}=(1-\nu)\left(1-\frac{\nu}{1-p}\right)^{-(1-p)},
$$

whence

$$
\nu=u_{1-p}\left(\frac{1}{\delta}\right) .
$$

Using relation $(25)$ and the notation $s=u_{p}(1 / \delta)$, we can rewrite this solution in the form

$$
\nu=\frac{(p-1) s}{p-s},
$$

which was used in the proof of Lemma 1.

We do not present independent estimations in the case of $p=\infty$, but simply pass to the limit in the expression for $\delta$ (formula (72)). As a result, we see that the " $A_{\infty}$-norm" of our weight is equal to $\delta=\frac{e^{-\nu}}{1-\nu}$.

I would like to thank S. V. Kislyakov who called my attention to the possibility of including the limit case where $p=1$ in the general framework; this case was absent in the initial version of the paper. 


\section{REFERENCES}

[1] R. R. Coifman and C. Fefferman, Weighted norm inequalities for maximum functions and singular integrals, Studia Math. 51 (1974), no. 3, 241-250. MR 50:10670

[2] F. Nazarov, S. Treil, and A. Volberg, Bellman function in stochastic control and harmonic analysis, Systems, Approximation, Singular Integral Operators, and Related Topics (Bordeaux, 2000), Oper. Theory Adv. Appl., vol. 129, Birkhäuser, Basel, 2001, pp. 393-423. MR 2003b:49024

[3] S. V. Khrushchev, A description of weights satisfying the $A_{\infty}$ condition of Muckenhoupt, Proc. Amer. Math. Soc. 90 (1984), 253-257. MR 85k:42049

[4] F. L. Nazarov and S. R. Treil, The hunt for a Bellman function: applications to estimates for singular integral operators and to other classical problems of harmonic analysis, Algebra i Analiz 8 (1996), no. 5, 32-162; English transl., St. Petersburg Math. J. 8 (1997), no. 5, 721-824. MR 99d:42026

[5] E. M. Stein, Harmonic analysis: real-variable methods, orthogonality, and oscillatory integrals, Princeton Math. Ser., vol. 43, Princeton Univ. Press, Princeton, NJ, 1993. MR 95c:42002

[6] E. M. Dyn'kin and B. P. Osilenker, Weighted estimates of singular integrals and their applications, Itogi Nauki i Tekhniki Mat. Anal., vol. 21, VINITI, Moscow, 1983, pp. 42-129; English transl., J. Soviet Math. 30 (1985), no. 3, 2094-2153. MR 85j:42034

St. Petersburg Branch, Steklov Mathematical Institute, Russian Academy of Sciences, Fontanka 27, St. Petersburg 191011, Russia

E-mail address: vasyunin@pdmi.ras.ru

Received 4/NOV/2002

Translated by THE AUTHOR 